\title{
Aggregation-Induced Emission and Organogels with Chiral and Racemic Pyrene substituted Cyanostyrenes
}

\author{
Palash Jana and Sriram Kanvah* \\ *Department of Chemistry, Indian Institute of Technology Gandhinagar, Palaj, Gujarat 382355, \\ E-mail:sriram@iitgn.ac.in, kanvah@gmail.com
}

\section{Supporting Information}

Characterization data of the synthesized compounds

Absorption, Emission spectra for the synthesized compounds

Cryo-SEM, drop-cast SEM, DLS data \& NMR-study

${ }^{1} \mathrm{H} \&{ }^{13} \mathrm{C}-\mathrm{NMR}$ spectra of synthesized compounds
$1-3$

4-7

$8-14$

$15-23$

\section{${ }^{1} \mathrm{H},{ }^{13} \mathrm{C}$ NMR Characterization data of the intermediate phenolic derivatives.}

Compound 7: Yield 70\% (400mg), Mp: 292-295 ${ }^{\circ} \mathrm{C}{ }^{1} \mathrm{H}-\mathrm{NMR}(500 \mathrm{MHz}, \mathrm{DMSO}-\mathrm{d} 6), \delta(\mathrm{ppm})$ $10.02(\mathrm{~s}, 1 \mathrm{H}), 8.82(\mathrm{~s}, 1 \mathrm{H}), 8.54-8.49(\mathrm{dd}, 2 \mathrm{H}), 8.43-8.39(\mathrm{~m}, 3 \mathrm{H}), 8.35-8.34(\mathrm{~d}, 1 \mathrm{H}), 8.31-8.25$ $(\mathrm{dd}, 3 \mathrm{H}), 8.17-8.14(\mathrm{t}, 1 \mathrm{H}), 7.83-7.81(\mathrm{~d}, 2 \mathrm{H}), 6.98-6.96(\mathrm{~d}, 2 \mathrm{H})$

${ }^{13} \mathrm{C}-\mathrm{NMR}(125 \mathrm{MHz}, \mathrm{DMSO}-\mathrm{d} 6): \delta$ (ppm), 159.35, 138.43, 132.26, 131.30, 130.85, 129.58, 129.38, 128.92, 128.87, 128.22, 127.79, 127.14, 126.70, 126.56, 126.42, 126.56, 126.70, 126.56, 126.42, 125.29, 125.10, 124.32, 124.16, 124.09, 118.65, 116.48, 115.11, HRMS (ESI-Q-TOF): $\mathrm{C}_{25} \mathrm{H}_{15} \mathrm{NO}[\mathrm{M}+\mathrm{H}]^{+}$: cal. $m / z 346.1226$, found, $m / z 346.1234$ (error $2.3 \mathrm{ppm}$ )

Compound 8: Yield 70\% (450mg): Mp: 273-276 ${ }^{\circ} \mathrm{C},{ }^{1} \mathrm{H}-\mathrm{NMR}$ (500MHz, DMSO-d6), $\delta(\mathrm{ppm})$ $9.55(\mathrm{~s}, 1 \mathrm{H}), 9.33(\mathrm{~s}, 1 \mathrm{H}), 8.72(\mathrm{~s}, 1 \mathrm{H}), 8.53-8.51(\mathrm{~d}, 2 \mathrm{H}), 8.48-8.46(\mathrm{~d}, 2 \mathrm{H}), 8.41-8.38(\mathrm{~m}, 2 \mathrm{H})$, 8.34-8.32 (d, 1H), 8.30-8.24 (dd, 2H), 8.16-8.13 (t, 1H), 7.37 (s,1H), 7.32-7.30 (dd, 1H), 6.94$6.93(\mathrm{~d}, 1 \mathrm{H})$

${ }^{13} \mathrm{C}-\mathrm{NMR}$ (125MHz, DMSO-d6): $\delta$ (ppm) 147.70, 146.30, 138.15, 132.22, 131.30, 129.55, 129.40, 128.87, 127.80, 127.14, 126.72, 126.56, 126.41, 125.56, 125.29, 124.32, 124.16, 124.04, $118.71,118.31,116.57,115.33,113.94$, HRMS (ESI-Q-TOF): $\mathrm{C}_{25} \mathrm{H}_{15} \mathrm{NO}_{2}[\mathrm{M}+\mathrm{H}]^{+}$: cal. $\mathrm{m} / \mathrm{z} 362.1176$, found, $\mathrm{m} / \mathrm{z} 362.1187$ (error 3ppm)

Compound 9: Yield 65\% (370mg): Mp: 250-255 ${ }^{\circ} \mathrm{C},{ }^{1} \mathrm{H}-\mathrm{NMR}(500 \mathrm{MHz}, \mathrm{DMSO}-\mathrm{d} 6), \delta(\mathrm{ppm})$ $9.28(\mathrm{~s}, 1 \mathrm{H}), 8.75(\mathrm{~s}, 1 \mathrm{H}), 8.64(\mathrm{~s}, 1 \mathrm{H}), 8.52-8.50(\mathrm{~d}, 1 \mathrm{H}), 8.46-8.39(\mathrm{~m}, 4 \mathrm{H}), 8.35-8.33(\mathrm{~d}, 1 \mathrm{H})$, $8.31-8.25(\mathrm{dd}, 2 \mathrm{H}), 8.17-8.14(\mathrm{t}, 1 \mathrm{H}), 6.94(\mathrm{~s}, 2 \mathrm{H})$ 
${ }^{13} \mathrm{C}-\mathrm{NMR}$ (125MHz, DMSO-d6): $\delta$ (ppm) 146.99, 137.99, 135.57, 132.20, 131.30, 129.51, $129.36,128.88,127.79,127.14,126.72,126.56,126.40,125.27,124.65,124.32,124.16,123.94$, 118.73, 115.58, 105.85. HRMS (ESI-Q-TOF): $\mathrm{C}_{25} \mathrm{H}_{15} \mathrm{NO}_{3}[\mathrm{M}+\mathrm{H}]^{+}$: cal. $m / z 378.1125$, found, $m / z 378.1134$ (error 2.4ppm)

\section{${ }^{1} \mathrm{H},{ }^{13} \mathrm{C}$ NMR Characterization data of the compounds}

Compound (1) MA: Yield 65\% (130mg): Yellow solid, m.p.: 105-110 ${ }^{\circ} \mathrm{C}, \mathrm{M}(\varepsilon)$ in $\mathrm{CH}_{3} \mathrm{CN}$ $=14449 \mathrm{M}^{-1} \mathrm{~cm}^{-1},{ }^{1} \mathrm{H}-\mathrm{NMR}\left(500 \mathrm{MHz}, \mathrm{CDCl}_{3}\right), \delta(\mathrm{ppm}): 8.63-8.62(\mathrm{~d}, 1 \mathrm{H}), 8.46(\mathrm{~s}, 1 \mathrm{H}), 8.29-8.26$ $(\mathrm{m}, 4 \mathrm{H}), 8.25-8.24(\mathrm{~d}, 1 \mathrm{H}), 8.23-8.15(\mathrm{dd}, 2 \mathrm{H}), 8.06-8.05(\mathrm{dd}, 1 \mathrm{H}), 7.78-7.76(\mathrm{~d}, 2 \mathrm{H}), 7.04-7.02$ $(\mathrm{d}, 2 \mathrm{H}), 5.14-5.11(\mathrm{t}, 1 \mathrm{H}), 4.11-4.07(\mathrm{~m}, 2 \mathrm{H}), 2.05-1.57(\mathrm{~m}, 16 \mathrm{H}), 1.43-1.41(\mathrm{~d}, 3 \mathrm{H})$

${ }^{13} \mathrm{C}-\mathrm{NMR}\left(125 \mathrm{MHz}, \mathrm{CDCl}_{3}\right): \delta(\mathrm{ppm}) 160.27,138.01,132.57,131.40,131.33,130.76,129.72$, $128.57,128.54,128.37,127.55,127.46,126.92,126.29,126.11,125.88,124.98,124.77,124.65$, 122.71, 118.34, 115.13, 114.63, 109.81, 66.63, 37.16, 36.09, 29.59, 25.74, 25.49, 19.60, 17.70, HRMS (ESI-Q-TOF): $\mathrm{C}_{35} \mathrm{H}_{34} \mathrm{NO}[\mathrm{M}+\mathrm{H}]^{+}$: cal. $\mathrm{m} / z 484.2635$, found, $m / z 484.2650$ (error 3ppm)

Compound (2) MC: Yield 65\% (130mg): Yellow solid, Mp: 105-110 ${ }^{\circ} \mathrm{C},(\varepsilon) \mathrm{CH}_{3} \mathrm{CN}=14449 \mathrm{M}^{-}$ ${ }^{1} \mathrm{~cm}^{-1}, 1 \mathrm{H}-\mathrm{NMR}\left(500 \mathrm{MHz}, \mathrm{CDCl}_{3}\right), \delta(\mathrm{ppm}): 8.63-8.62(\mathrm{~d}, 1 \mathrm{H}), 8.46(\mathrm{~s}, 1 \mathrm{H}), 8.29-8.26(\mathrm{~m}, 4 \mathrm{H})$, 8.25-8.24 (d, $1 \mathrm{H}), 8.23-8.15(\mathrm{dd}, 2 \mathrm{H}), 8.06-8.03(\mathrm{dd}, 1 \mathrm{H}), 7.78-7.76(\mathrm{~d}, 2 \mathrm{H}), 7.04-7.02(\mathrm{~d}, 2 \mathrm{H})$, 5.14-5.11(t, 1H), 4.11-4.07 (m, 2H), 2.05-1.57 (m, 16H), 1.43-1.41 (d, 3H)

${ }^{13} \mathrm{C}-\mathrm{NMR}\left(125 \mathrm{MHz}, \mathrm{CDCl}_{3}\right): \delta$ (ppm) 160.27, 138.01, 132.57, 131.40, 131.33, 130.76, 129.72, $128.57,128.54,128.37,127.55,127.46,126.92,126.29,126.11,125.88,124.98,124.77,124.65$, 122.71, 118.34, 115.13, 114.63, 109.81, 66.63, 37.16, 36.09, 29.59, 25.74, 25.49, 19.60, 17.70, HRMS (ESI-Q-TOF): $\mathrm{C}_{35} \mathrm{H}_{34} \mathrm{NO}[\mathrm{M}+\mathrm{H}]^{+}$: cal. $\mathrm{m} / z 484.2635$, found, $\mathrm{m} / \mathrm{z} 484.2650$ (error 3ppm)

Compound (3) DA: Yellow semi-solid, $\varepsilon\left(\mathrm{CH}_{3} \mathrm{CN}\right)=15596 \mathrm{M}^{-1} \mathrm{~cm}^{-1}$, Yield 55\% $(120 \mathrm{mg}),{ }^{1} \mathrm{H}-$ NMR $\left(500 \mathrm{MHz}, \mathrm{CDCl}_{3}\right) \delta(\mathrm{ppm}): 8.61-8.60(\mathrm{~d}, 1 \mathrm{H}), 8.44(\mathrm{~s}, 1 \mathrm{H}), 8.28-8.23(\mathrm{dd}, 4 \mathrm{H}) 8.19-8.17(\mathrm{~d}$, 1H) 8.15-8.09 (dd, $2 \mathrm{H}), 8.13-8.09(\mathrm{dd}, 1 \mathrm{H}), 7.41-7.39(\mathrm{dd}, 1 \mathrm{H}), 7.33(\mathrm{~s}, 1 \mathrm{H}), 7.00-6.98(\mathrm{~d}, 1 \mathrm{H})$, 5.13-5.11 (m, 2H), 4.16-4.11 (m, 4H), 2.17-1.25 (m, 23H), 1.01-0.99 (d, 6H)

${ }^{13} \mathrm{C}-\mathrm{NMR}\left(125 \mathrm{MHz}, \mathrm{CDCl}_{3}\right): \delta$ (ppm) 150.61, 149.53, 138.35, 132.60, 131.35, 131.31, 131.84, $130.78,129.73,128.60,128.56,127.48,127.42,126.32,126.21,126.13,125.90,125.00,124.79$, $124.73,124.70,124.65,122.78,119.45,118.33,114.95,113.59,111.68,68.00,67.70,37.27$, $37.23,36.35,36.11,29.75,29.73,25.72,25.70,25.55,19.65,19.63,17.66$, HRMS (ESI-Q-TOF): $\mathrm{C}_{45} \mathrm{H}_{52} \mathrm{NO}_{2}[\mathrm{M}+\mathrm{H}]^{+}$: cal. $m / z$ 638.3993, found, $m / z 638.4020$ (error 4ppm)

Compound (4) DC: Yellow semi-solid, ( $(\varepsilon)$ in $\mathrm{CH}_{3} \mathrm{CN}=15596 \mathrm{M}^{-1} \mathrm{~cm}^{-1}$, Yield $60 \%(120 \mathrm{mg}):{ }^{1} \mathrm{H}-$ NMR (500MHz, $\left.\mathrm{CDCl}_{3}\right) \delta(\mathrm{ppm}): 8.61-8.60(\mathrm{~d}, 1 \mathrm{H}), 8.44(\mathrm{~s}, 1 \mathrm{H}), 8.28-8.23(\mathrm{dd}, 4 \mathrm{H})$ 8.19-8.17 
(d, 1H) 8.15-8.09 (dd, 2H), 8.13-8.09 (dd, 1H), 7.41-7.39 (dd, 1H), $7.33(\mathrm{~s}, 1 \mathrm{H}), 7.00-6.98(\mathrm{~d}$, $1 \mathrm{H}), 5.13-5.11(\mathrm{~m}, 2 \mathrm{H}), 4.16-4.11(\mathrm{~m}, 4 \mathrm{H}), 2.17-1.25(\mathrm{~m}, 23 \mathrm{H}), 1.01-0.99(\mathrm{~d}, 6 \mathrm{H})$

${ }^{13} \mathrm{C}-\mathrm{NMR}\left(125 \mathrm{MHz}, \mathrm{CDCl}_{3}\right.$ ): $\delta$ (ppm) 150.61, 149.53, 138.35, 132.60, 131.35, 131.31, 131.84, $130.78,129.73,128.60,128.56,127.48,127.42,126.32,126.21,126.13,125.90,125.00,124.79$, $124.73,124.70,124.65,122.78,119.45,118.33,114.95,113.59,111.68,68.00,67.70,37.27$, 37.23, 36.35, 36.11, 29.75, 29.73, 25.72, 25.70, 25.55, 19.65, 19.63, 17.66, HRMS (ESI-Q-TOF): $\mathrm{C}_{45} \mathrm{H}_{52} \mathrm{NO}_{2}[\mathrm{M}+\mathrm{H}]^{+}$: cal. $m / z$ 638.3993, found, $m / z 638.4020$ (error 4ppm)

Compound (5) TA: Yellow liquid, $(\varepsilon)$ in $\mathrm{CH}_{3} \mathrm{CN}=10621 \mathrm{M}^{-1} \mathrm{~cm}^{-1}$, Yield $50 \%(159 \mathrm{mg}):{ }^{1} \mathrm{H}-$ NMR (500MHz, $\left.\mathrm{CDCl}_{3}\right), \delta(\mathrm{ppm}): 8.60-8.59(\mathrm{~d}, 1 \mathrm{H}), 8.4(\mathrm{~s}, 1 \mathrm{H}), 8.27-8.24(\mathrm{~m}, 4 \mathrm{H}), 8.20-8.18$ $(\mathrm{d}, 1 \mathrm{H}), 8.18-8.16(\mathrm{dd}, 2 \mathrm{H}), 8.12-8.05(\mathrm{dd}, 1 \mathrm{H}), 7.01(\mathrm{~s}, 2 \mathrm{H}), 5.13-5.11(\mathrm{~m}, 3 \mathrm{H}), 4.15-4.0(\mathrm{~m}, 6 \mathrm{H})$, 2.04-1.22 (m,48H) 0.99-0.97 (t, 9H),

${ }^{13} \mathrm{C}-\mathrm{NMR}\left(125 \mathrm{MHz}, \mathrm{CDCl}_{3}\right): \delta$ (ppm) 153.64, 139.72, 139.61, 132.75, 131.34, 131.23, 131.09, $130.77,129.79,129.76,128.70,128.31,127.48,126.36,126.24,126.22,125.99,135.02,124.91$, $124.78,124.69,124.62,122.76,118.27,115.24,105.13,71.87,67.81,37.39,37.28,37.25,37.23$, 29.62, 29.52, 29.34, 25.71, 25.69, 25.38, 19.57, 19.54, 19.51, 17.67, 17.62, HRMS (ESI-Q-TOF): $\mathrm{C}_{55} \mathrm{H}_{70} \mathrm{NO}_{3}[\mathrm{M}+\mathrm{H}]^{+}$: cal. $m / z 792.5350$, found, $m / z 792.5375$ (error 3.1ppm)

Compound (6) TC: Yellow liquid, ( $(\varepsilon)$ in $\mathrm{CH}_{3} \mathrm{CN}=10621 \mathrm{M}^{-1} \mathrm{~cm}^{-1}$, Yield $50 \%$ (159mg): ${ }^{1} \mathrm{H}-\mathrm{NMR}$ $\left(500 \mathrm{MHz}, \mathrm{CDCl}_{3}\right), \delta(\mathrm{ppm}): 8.60-8.59(\mathrm{~d}, 1 \mathrm{H}), 8.4(\mathrm{~s}, 1 \mathrm{H}), 8.27-8.24(\mathrm{~m}, 4 \mathrm{H}), 8.20-8.18(\mathrm{~d}, 1 \mathrm{H})$, 8.18-8.16 (dd, $2 \mathrm{H}), 8.12-8.05(\mathrm{dd}, 1 \mathrm{H}), 7.01(\mathrm{~s}, 2 \mathrm{H}), 5.13-5.11(\mathrm{~m}, 3 \mathrm{H}), 4.15-4.0(\mathrm{~m}, 6 \mathrm{H}), 2.04-$ $1.22(\mathrm{~m}, 48 \mathrm{H}) 0.99-0.97(\mathrm{t}, 9 \mathrm{H})$

${ }^{13} \mathrm{C}-\mathrm{NMR}\left(125 \mathrm{MHz}, \mathrm{CDCl}_{3}\right): \delta$ (ppm) 153.64, 139.72, 139.61, 132.75, 131.34, 131.23, 131.09, $130.77,129.79,129.76,128.70,128.31,127.48,126.36,126.24,126.22,125.99,135.02,124.91$, $124.78,124.69,124.62,122.76,118.27,115.24,105.13,71.87,67.81,37.39,37.28,37.25,37.23$, 29.62, 29.52, 29.34, 25.71, 25.69, 25.38, 19.57, 19.54, 19.51, 17.67, 17.62, HRMS (ESI-Q-TOF): $\mathrm{C}_{55} \mathrm{H}_{70} \mathrm{NO}_{3}[\mathrm{M}+\mathrm{H}]^{+}$: cal. $m / z 792.5350$, found, $m / z 792.5375$ (error 3.1ppm) 

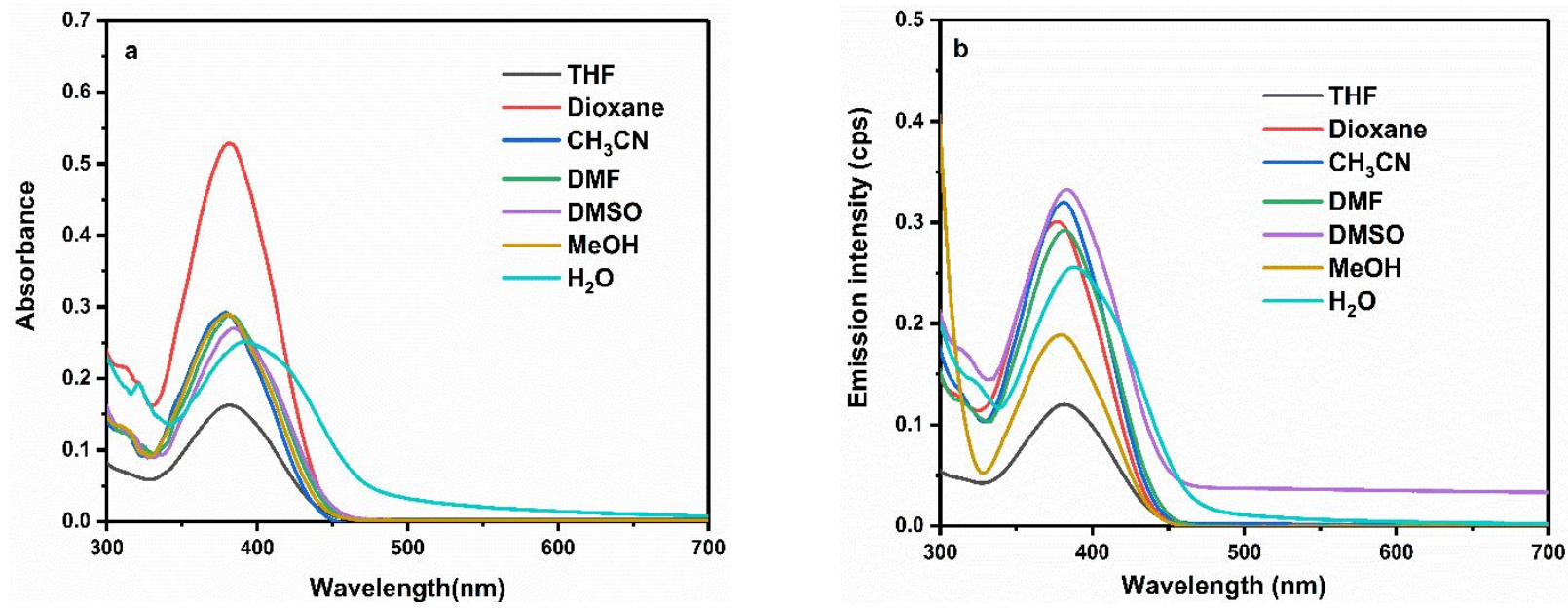

Fig.S1 Absorption spectra of a) MA b) MC in different organic solvents and water. Concentration $20 \mu \mathrm{M}$, ExW1 385nm
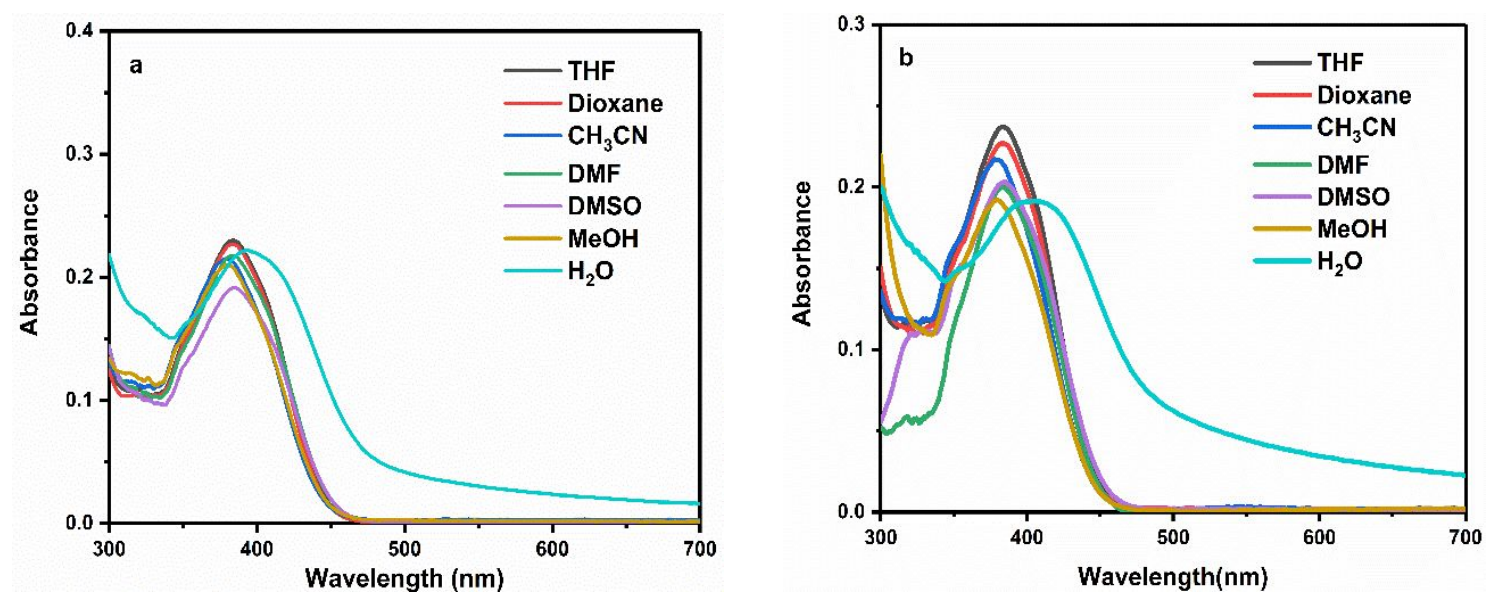

Fig.S2 Absorption spectra of a) TA b) TC in different organic solvents and water. Concentration $20 \mu \mathrm{M}$, ExW1. 385nm 

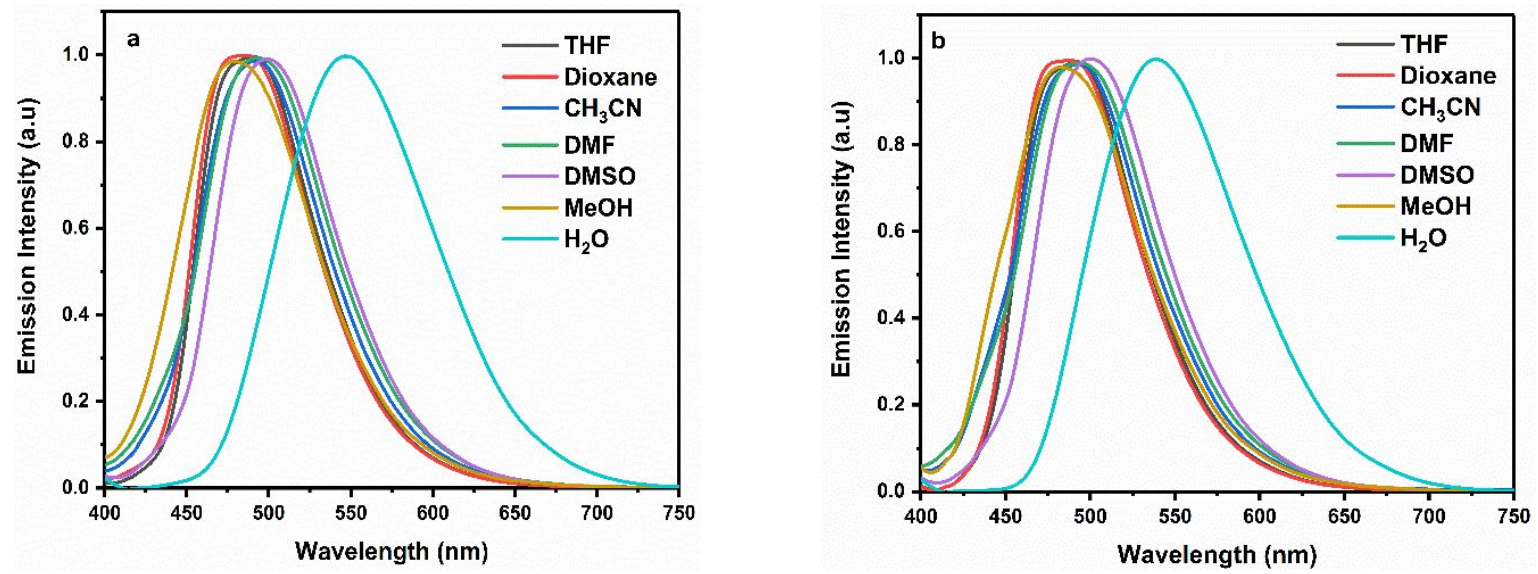

Fig S3 Normalized Emission spectra of a) DA b) DC in different organic solvents and water. Concentration $20 \mu \mathrm{M}$, ExW1 385nm
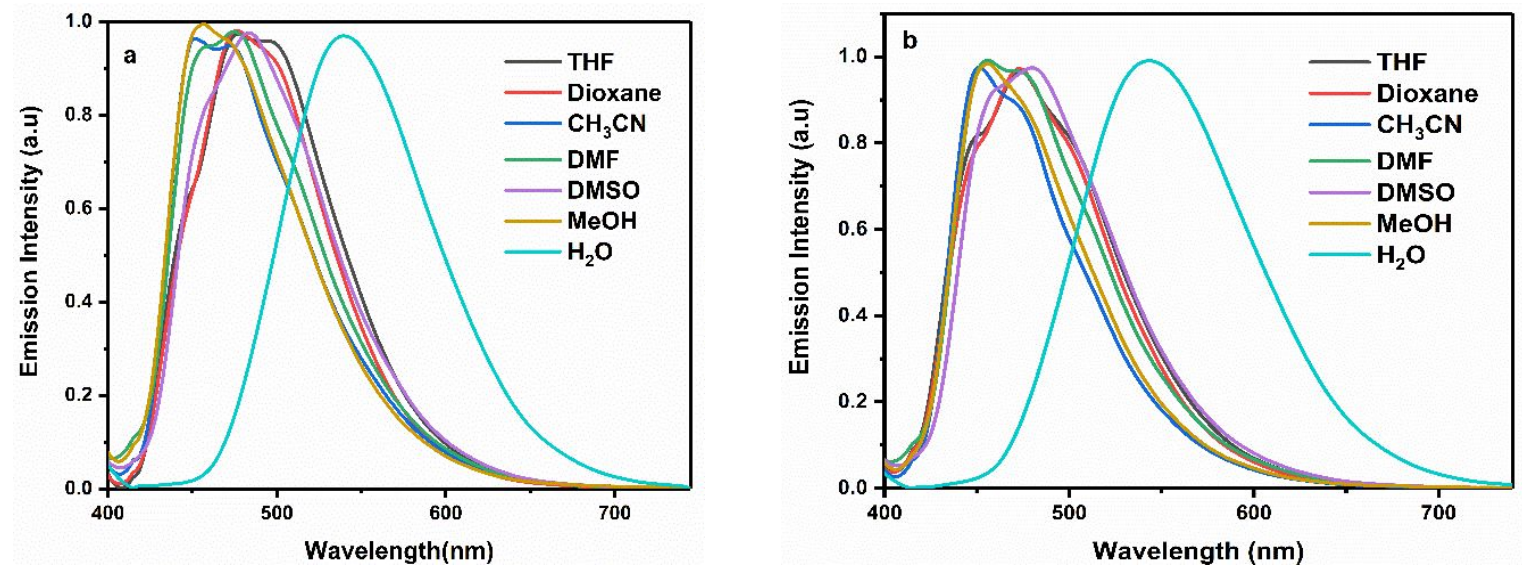

Fig.S4 Normalized Emission spectra of a) TA b) TC in different organic solvents and water. Concentration $20 \mu \mathrm{M}$, ExW1 385nm 

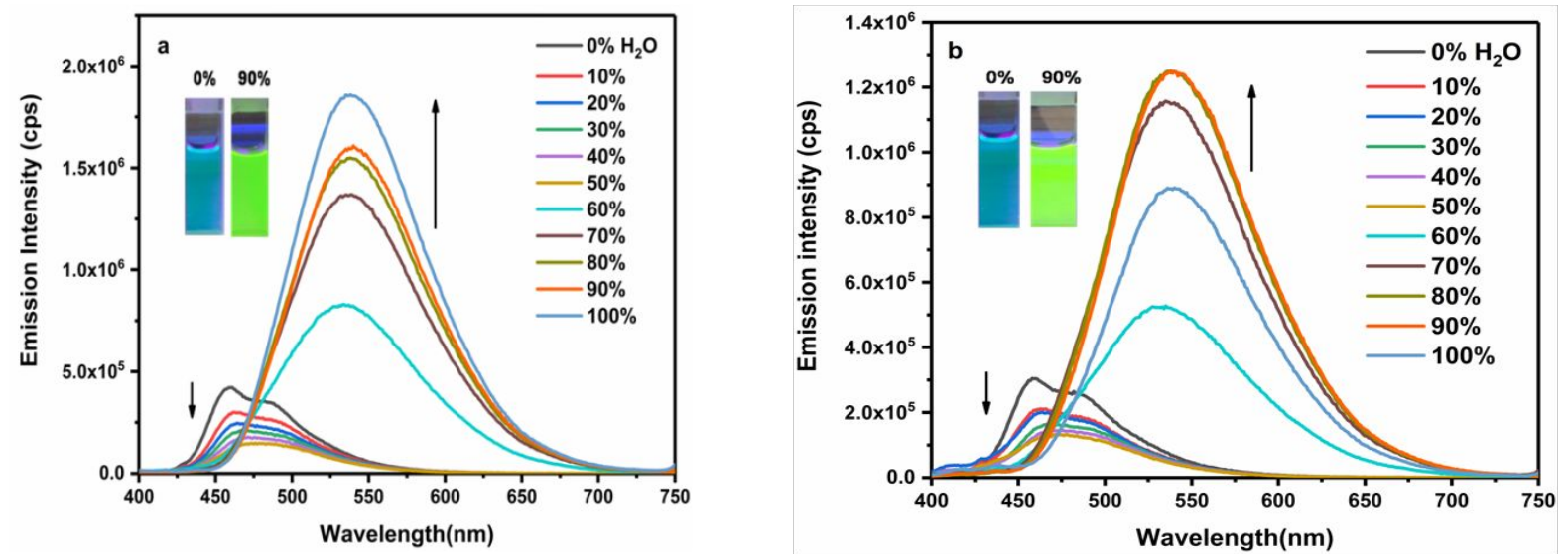

Fig.S5 Emission spectra of a) MA b) MC in water/binary mixture. Concentration $20 \mu \mathrm{M}$, ExWl $380 \mathrm{~nm}$
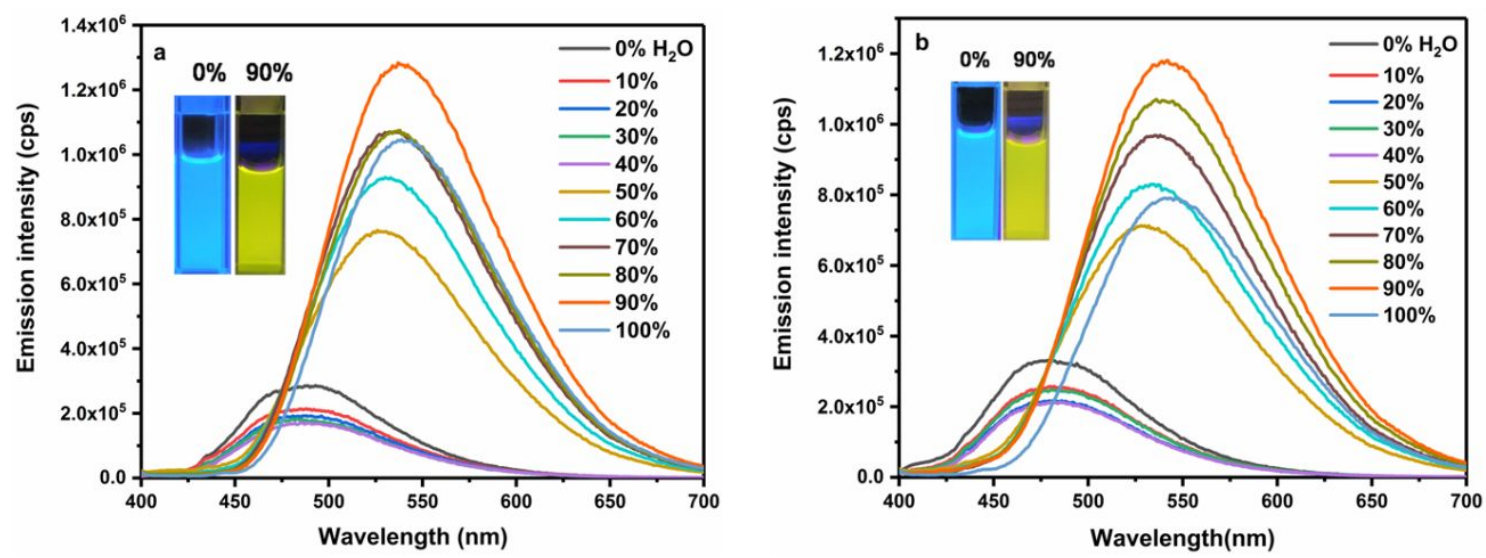

Fig.S6 Emission spectra of a) TA b) TC in water/binary mixture . Concentration $20 \mu \mathrm{M}$, ExWl $385 \mathrm{~nm}$
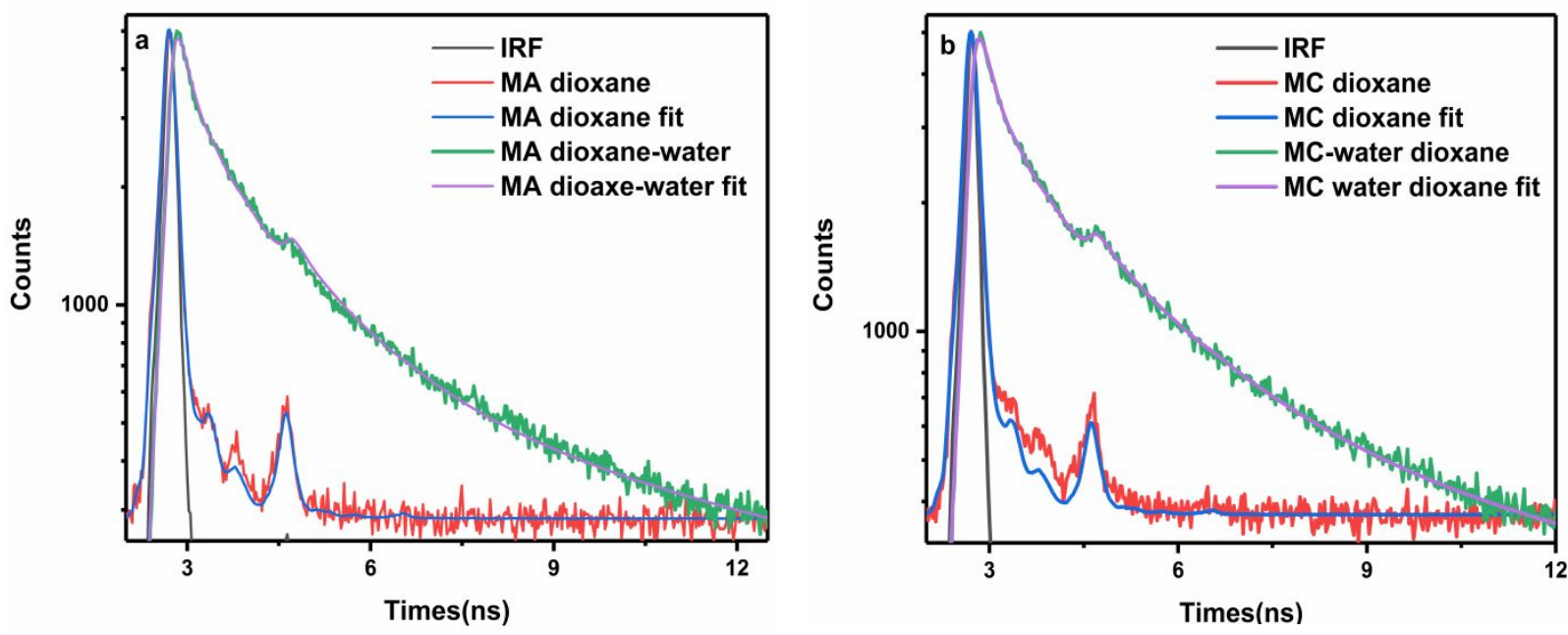

Fig.S7 Lifetime decay of a) MA \& b) MC in $90 \%$ water solution at $405 \mathrm{~nm}$ excitation 

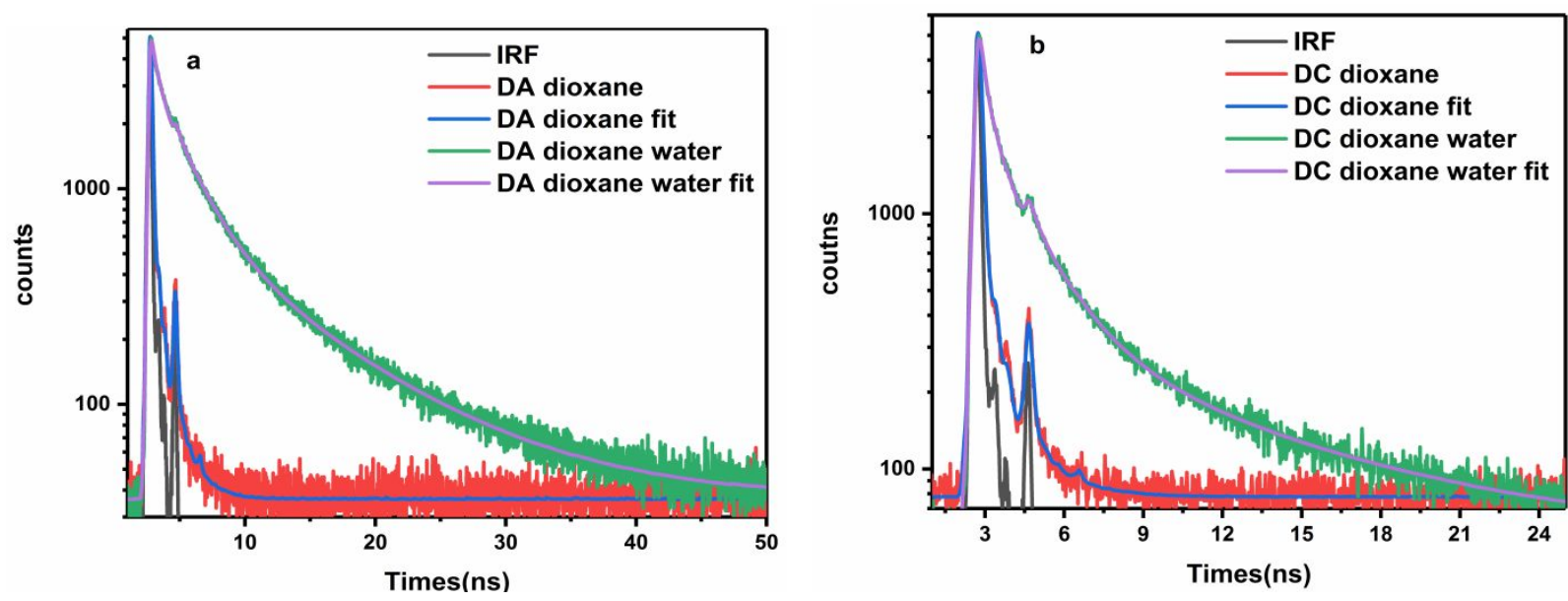

Fig.S8 Lifetime decay of a) DA \& b) DC in $90 \%$ water solution at $405 \mathrm{~nm}$ excitation
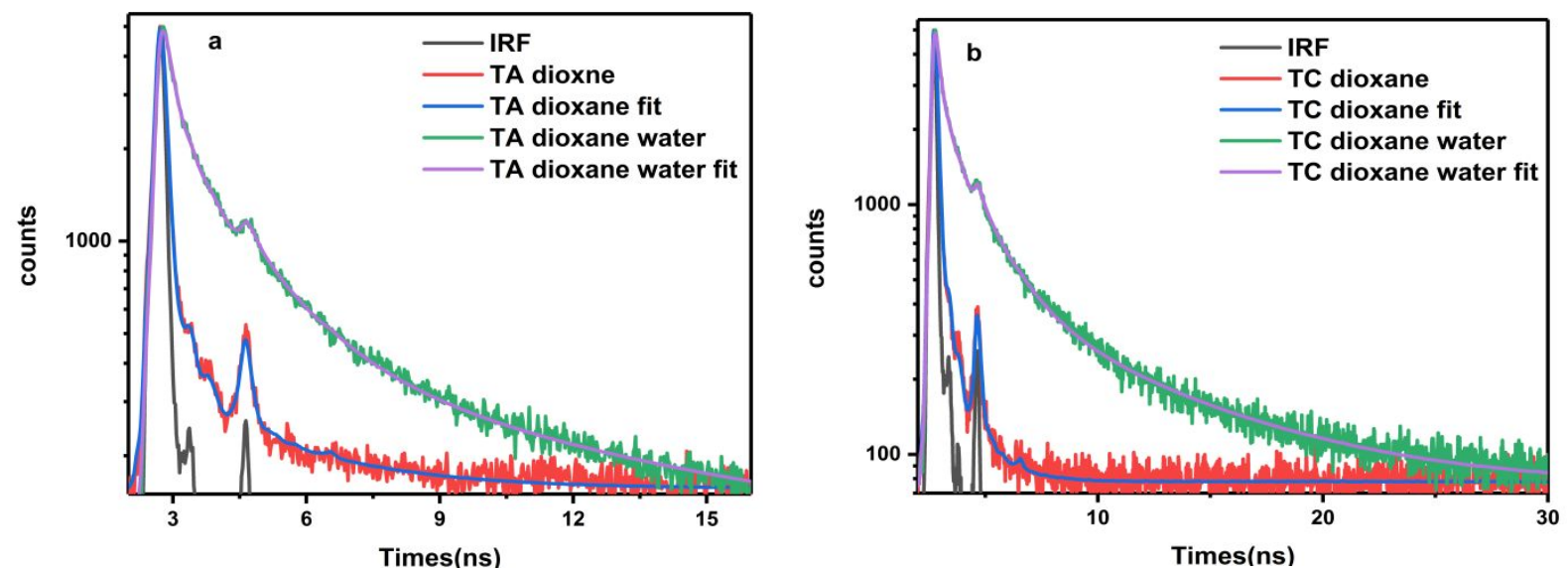

Fig.S9 Lifetime decay of a) TA \& b) TC in $90 \%$ water solution at $405 \mathrm{~nm}$ excitation. 


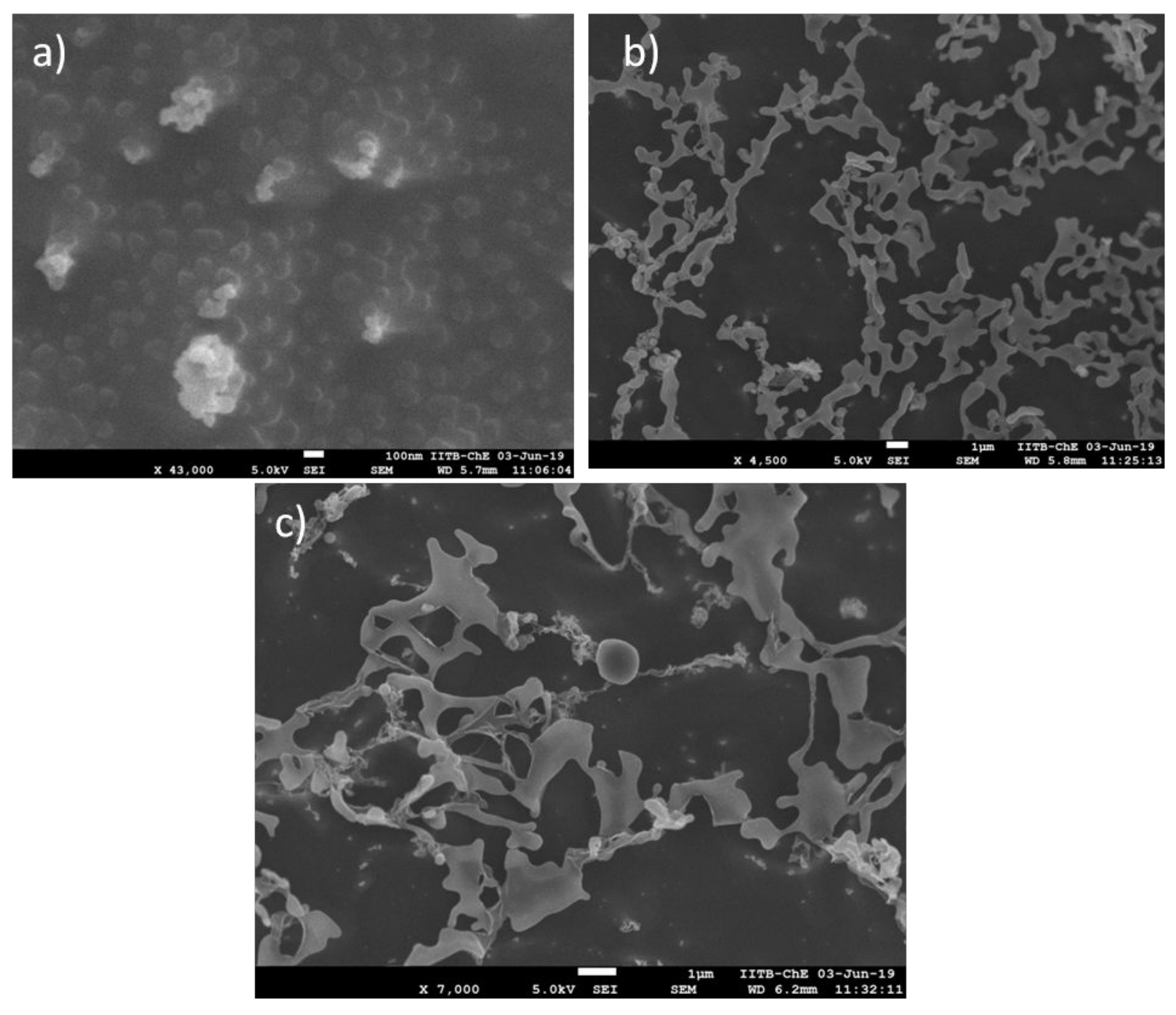

Fig.S10 Cryo-SEM images of MC, DC and TC. 

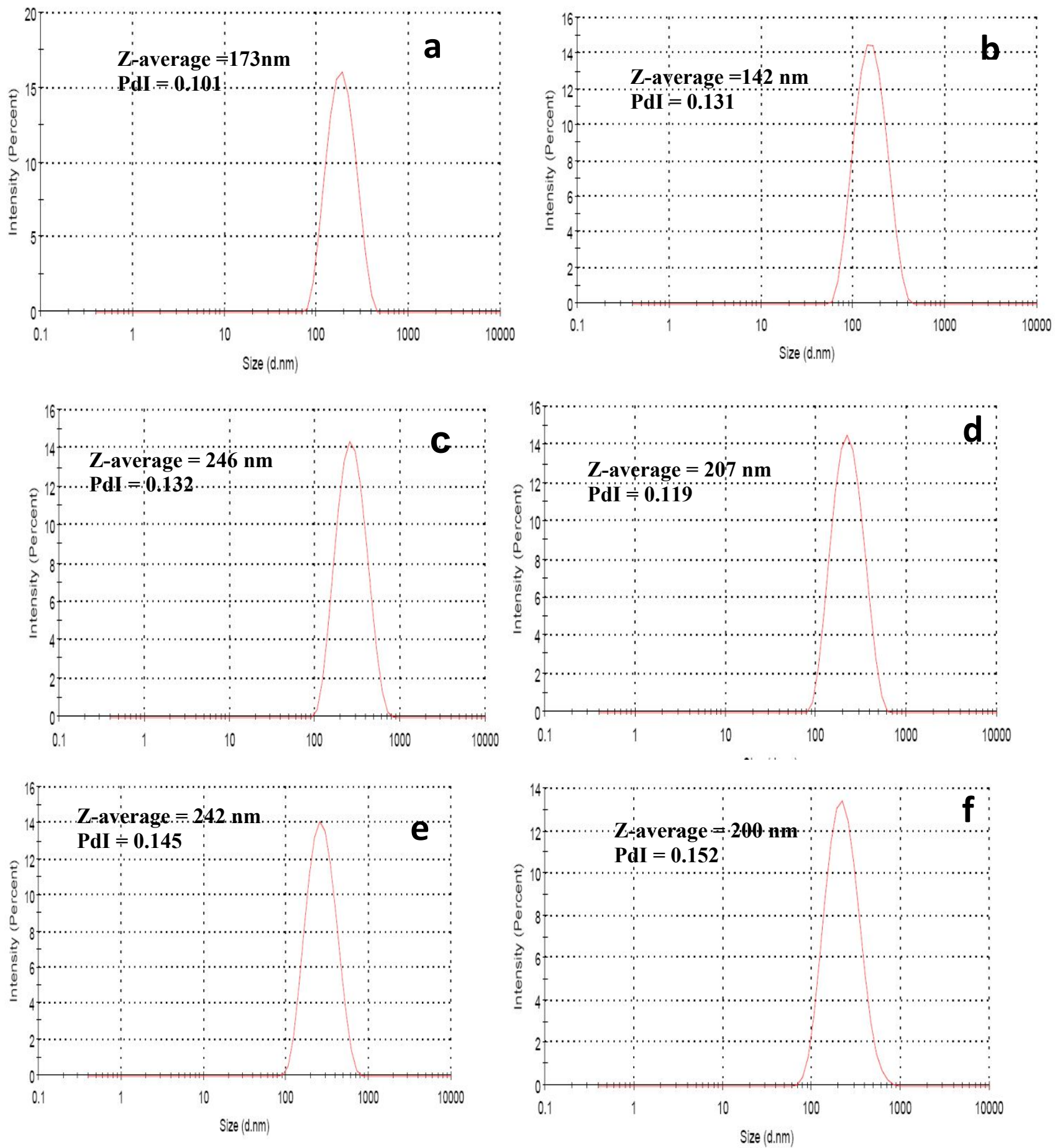

Fig.S11 DLS data of a) MA, b) MC, c) DA, d) DC, e) TA, and f) TC. 

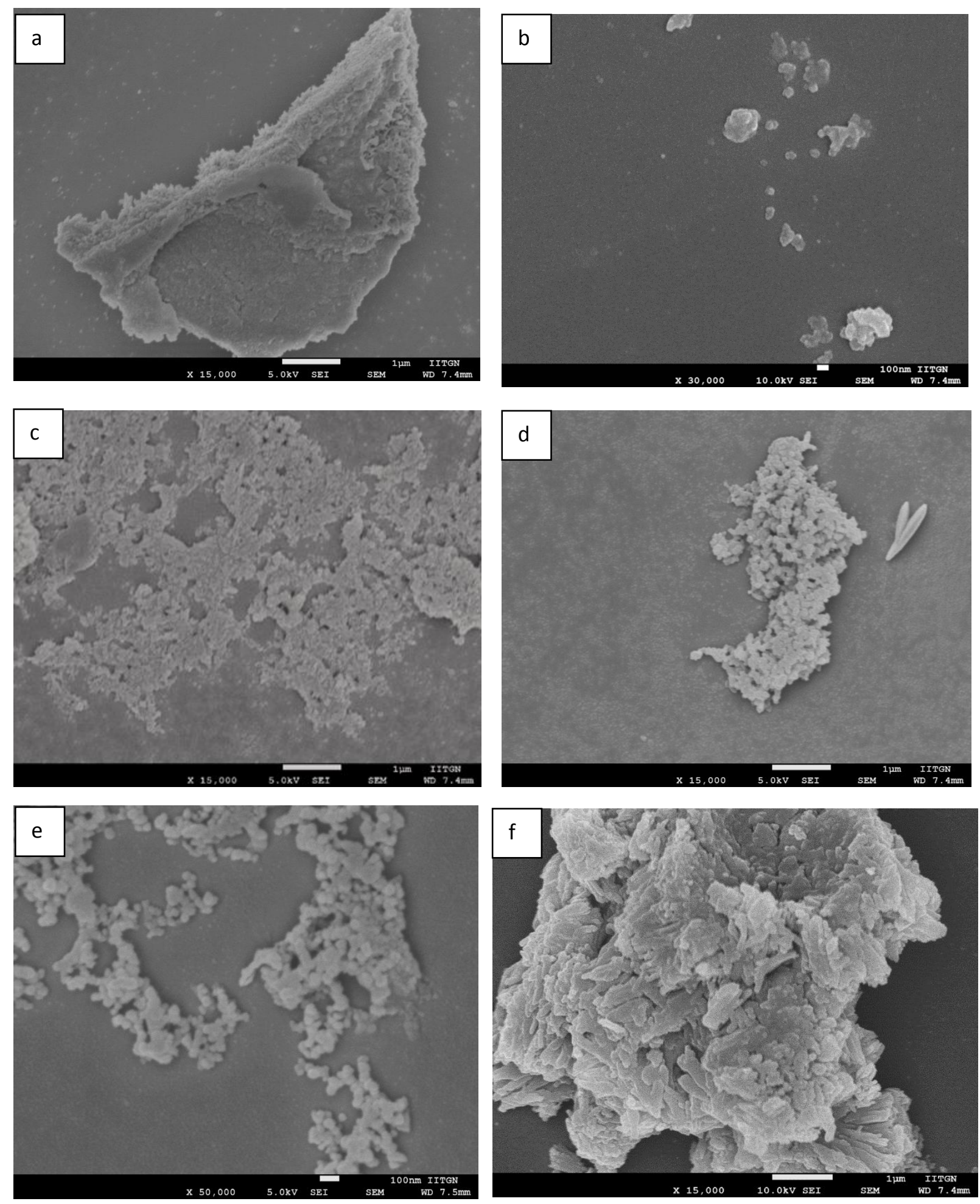

Fig S12: Dropcast SEM images obtained for a) MA, MC b) DA, DC and c) TA and TC 

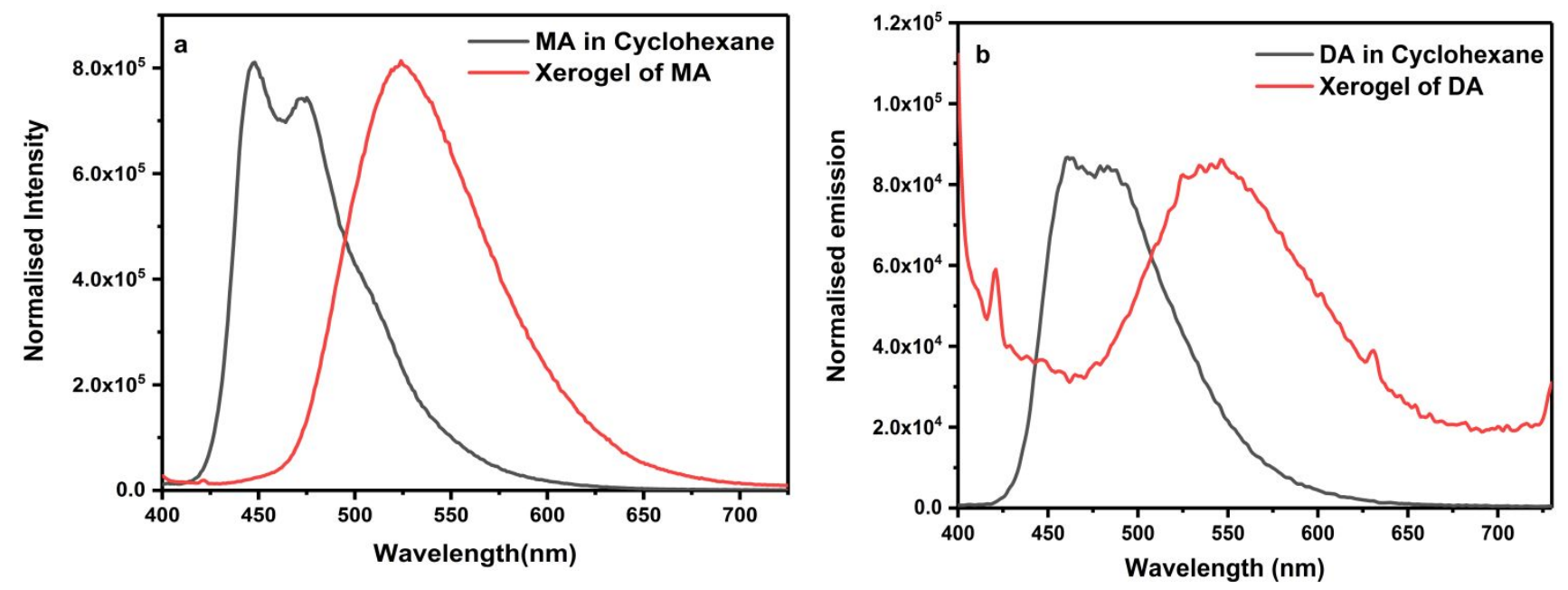

Fig.S13 The emission of organogels of a) MA and b) DA and their solution state.

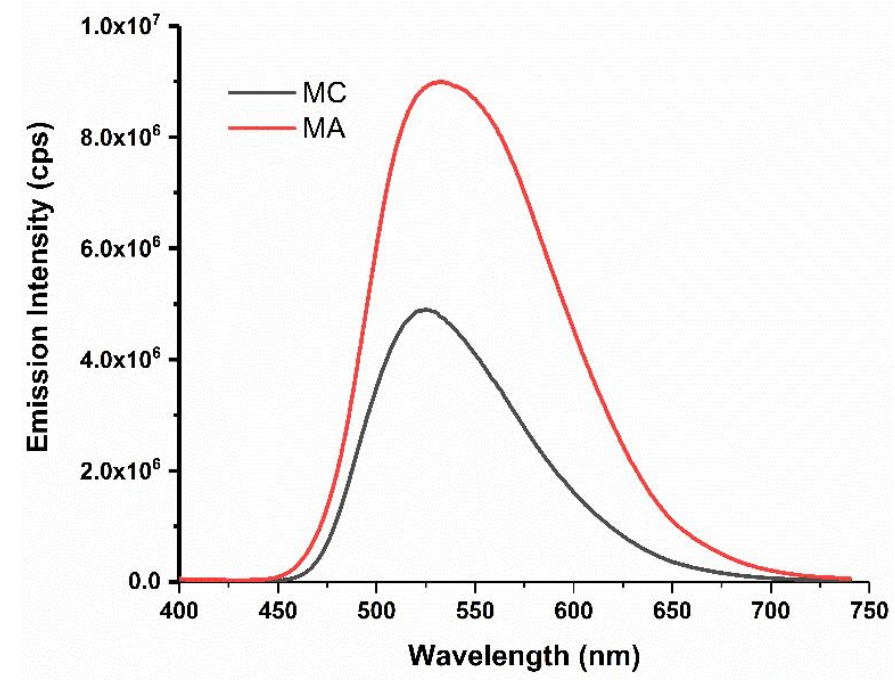

Fig.S14 The emission spectra of MA \& MC in solid state.Ex.W1 385nm 

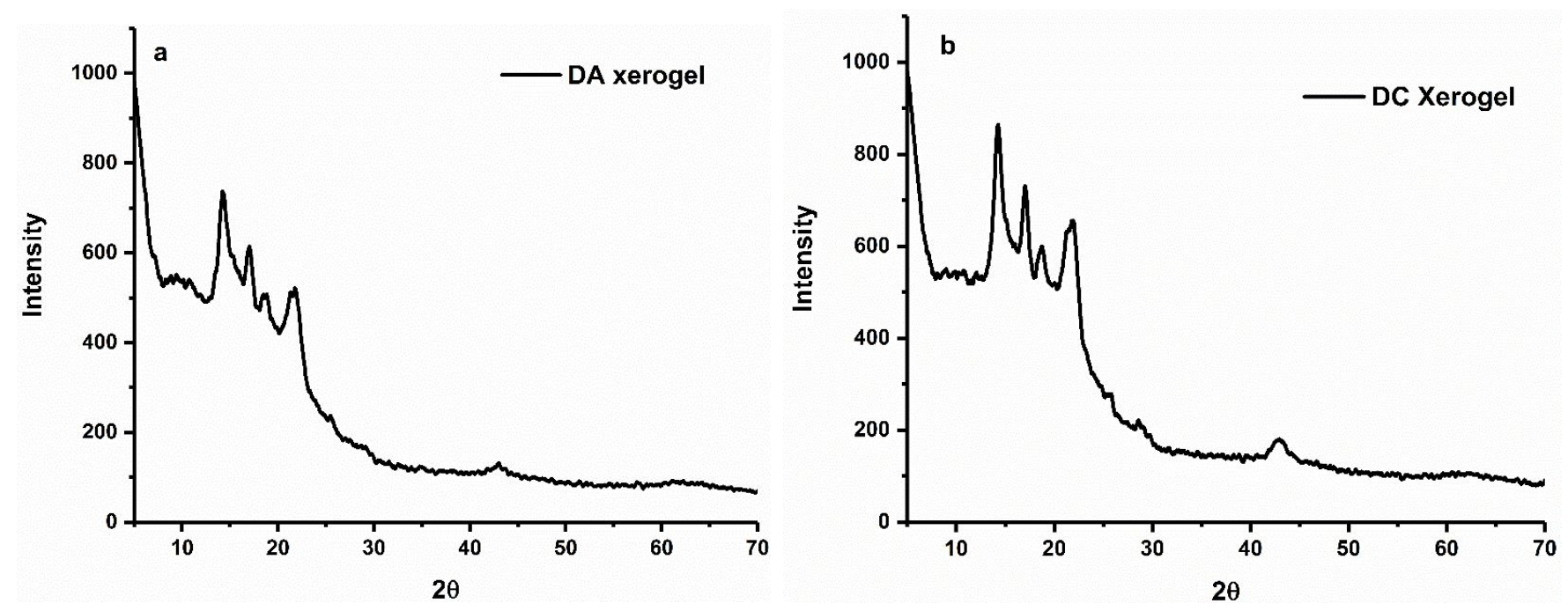

Fig.S15 Powder XRD data of DA \& DC obtained in its xerogel state. The compounds are semisolids and hence powdered measurement was not performed.

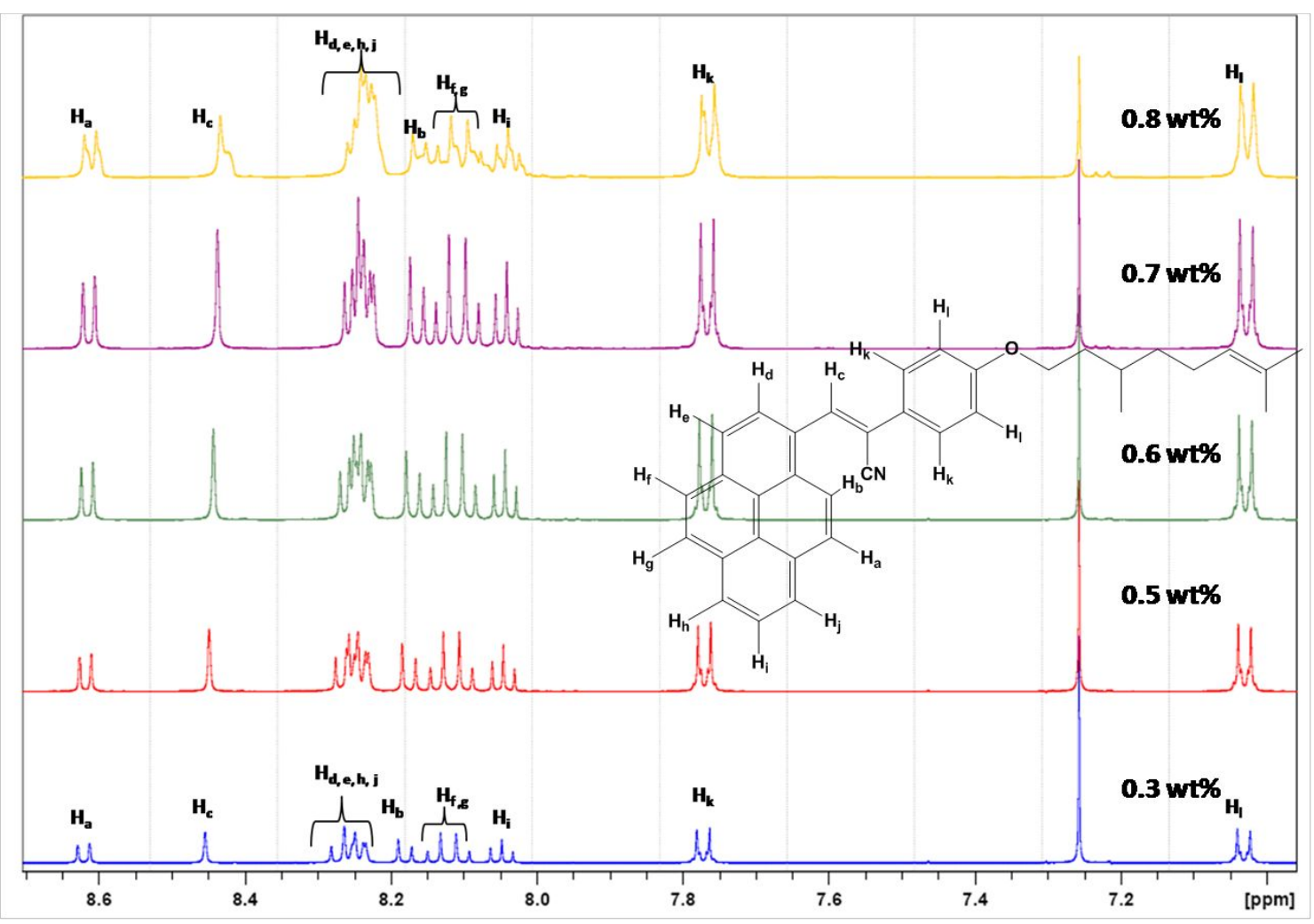

Fig. $\mathrm{S} 16{ }^{1} \mathrm{H}-\mathrm{NMR}$ titration spectra of $\mathrm{MA}$ in $\mathrm{CDCl}_{3}$ solution (aromatic region) 


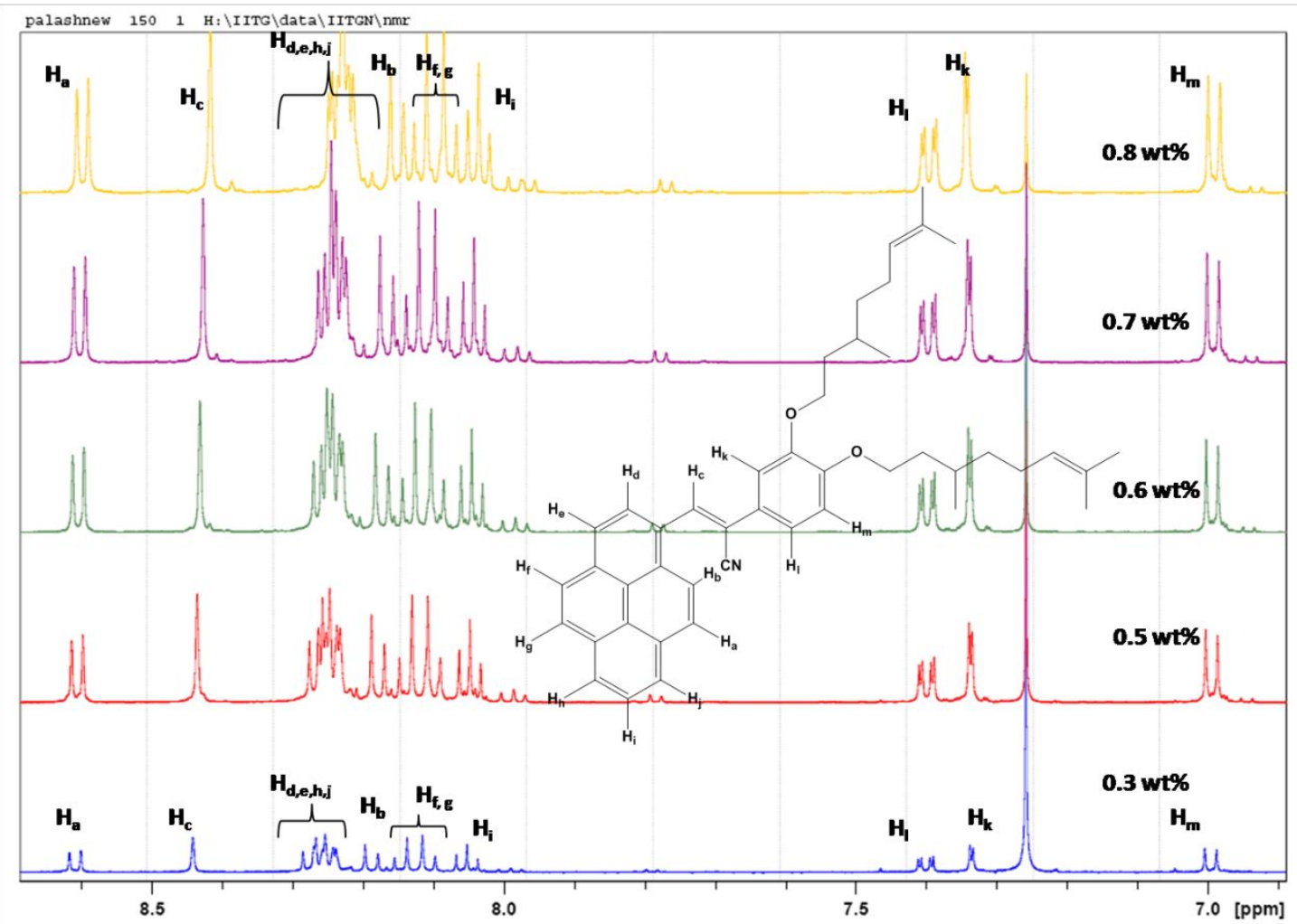

Fig.S17 ${ }^{1} \mathrm{H}-\mathrm{NMR}$ titration spectra of $\mathrm{DA}$ in $\mathrm{CDCl}_{3}$ solution (aromatic region)

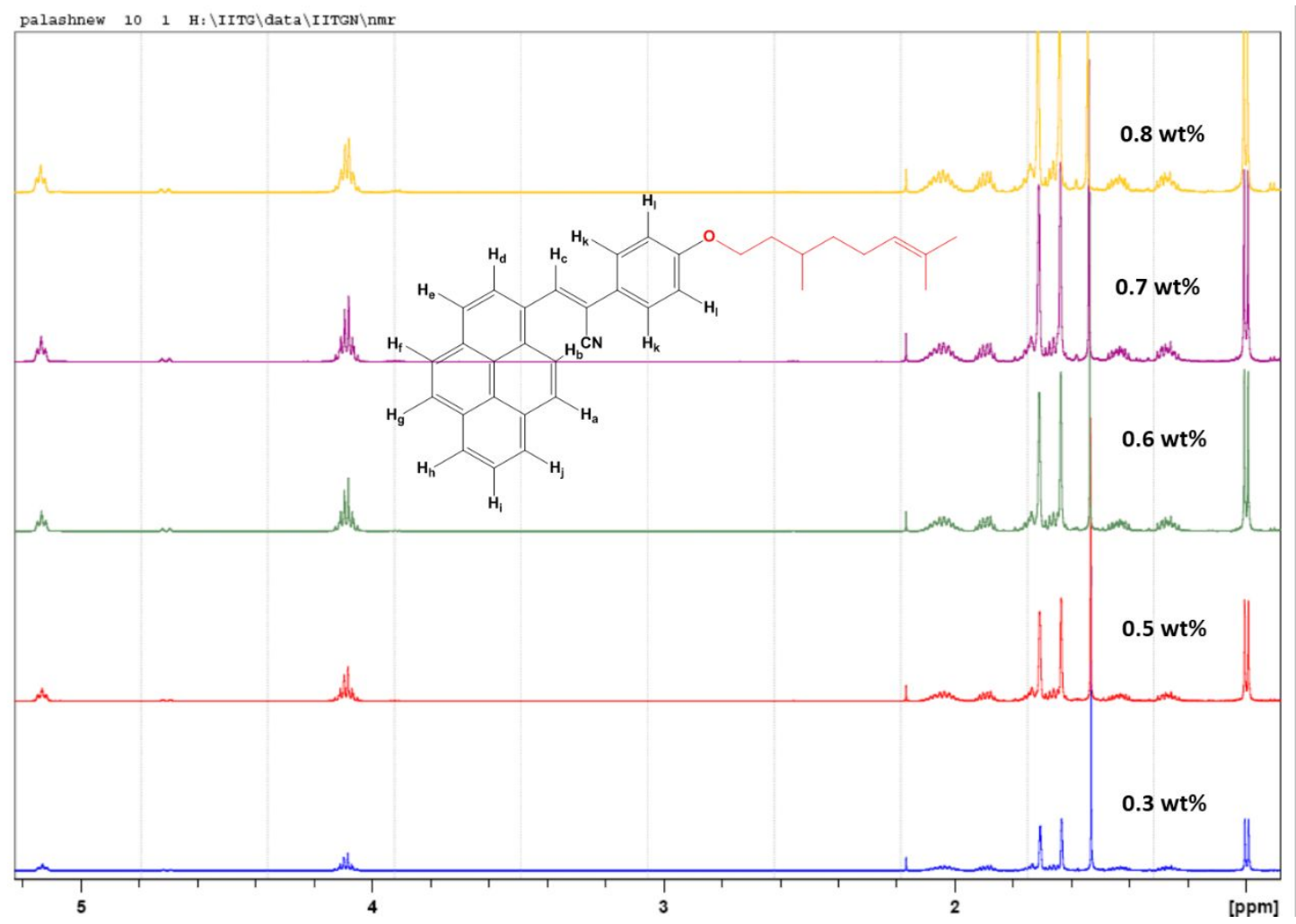

Fig.S18 ${ }^{1} \mathrm{H}-\mathrm{NMR}$ titration spectra of $\mathrm{MA}$ in $\mathrm{CDCl}_{3}$ solution (aliphatic region) 


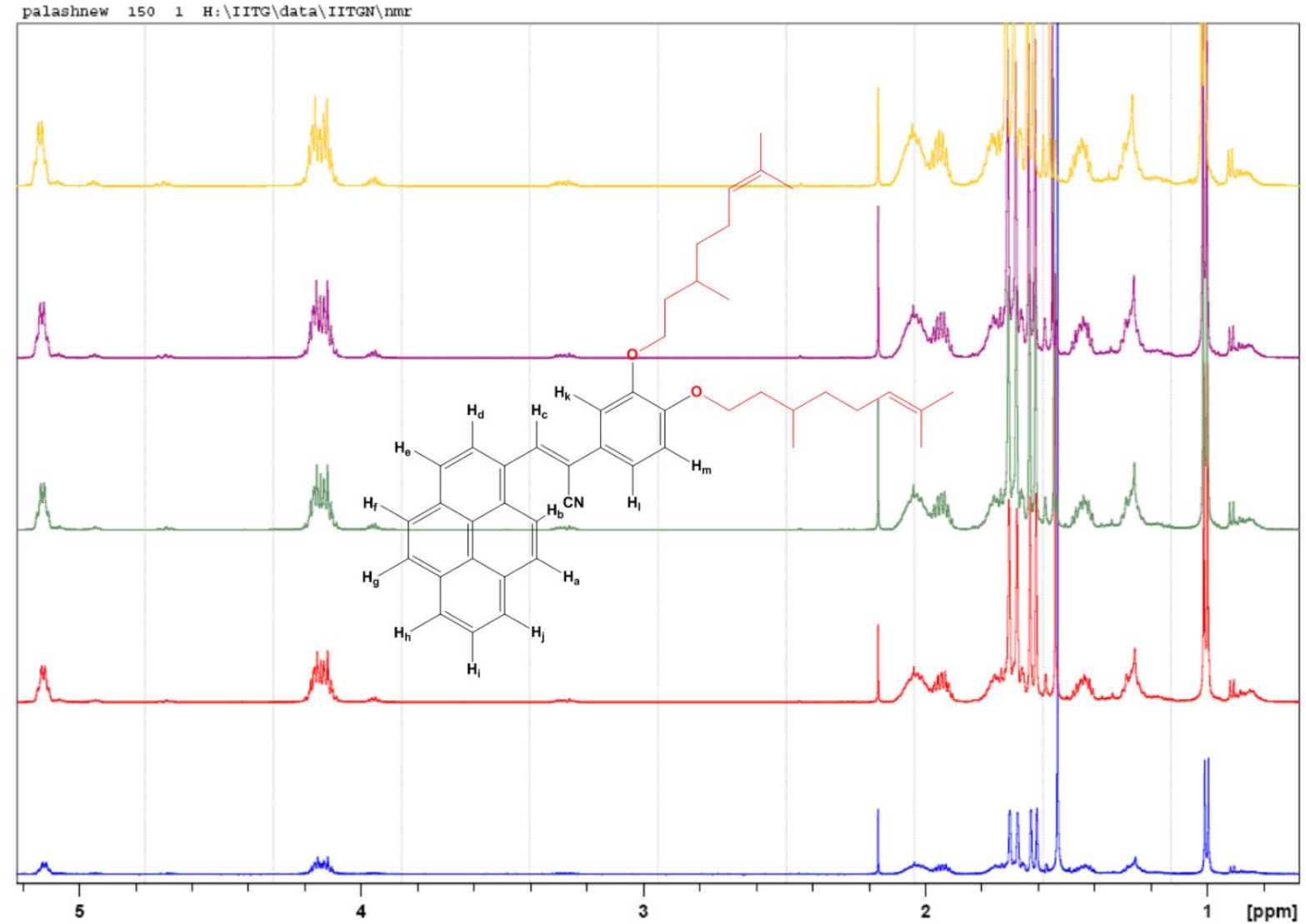

Fig.S19 ${ }^{1} \mathrm{H}-\mathrm{NMR}$ titration spectra of DA in $\mathrm{CDCl}_{3}$ solution (aliphatic region) 


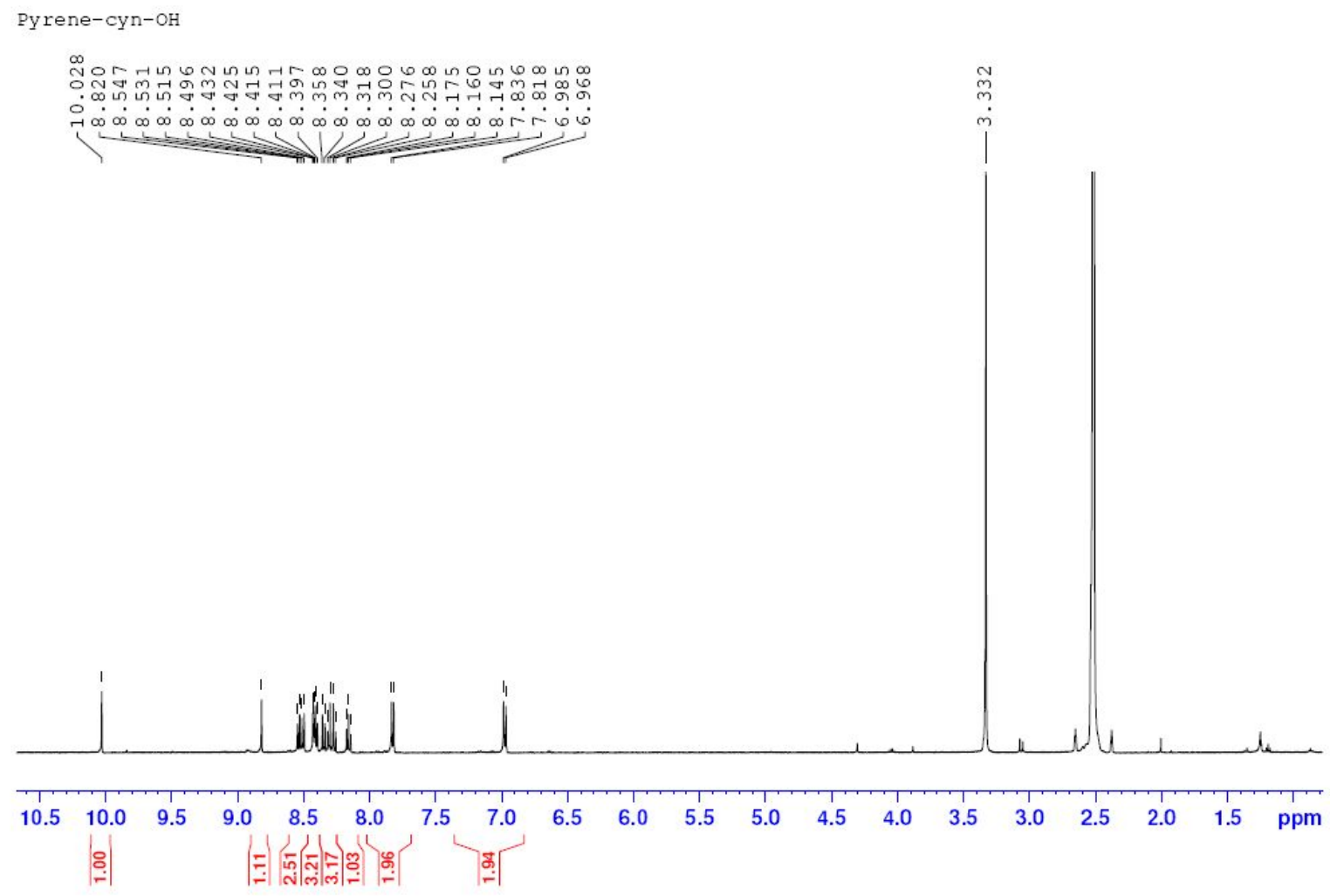

Pyrene-cyn-OH 13C
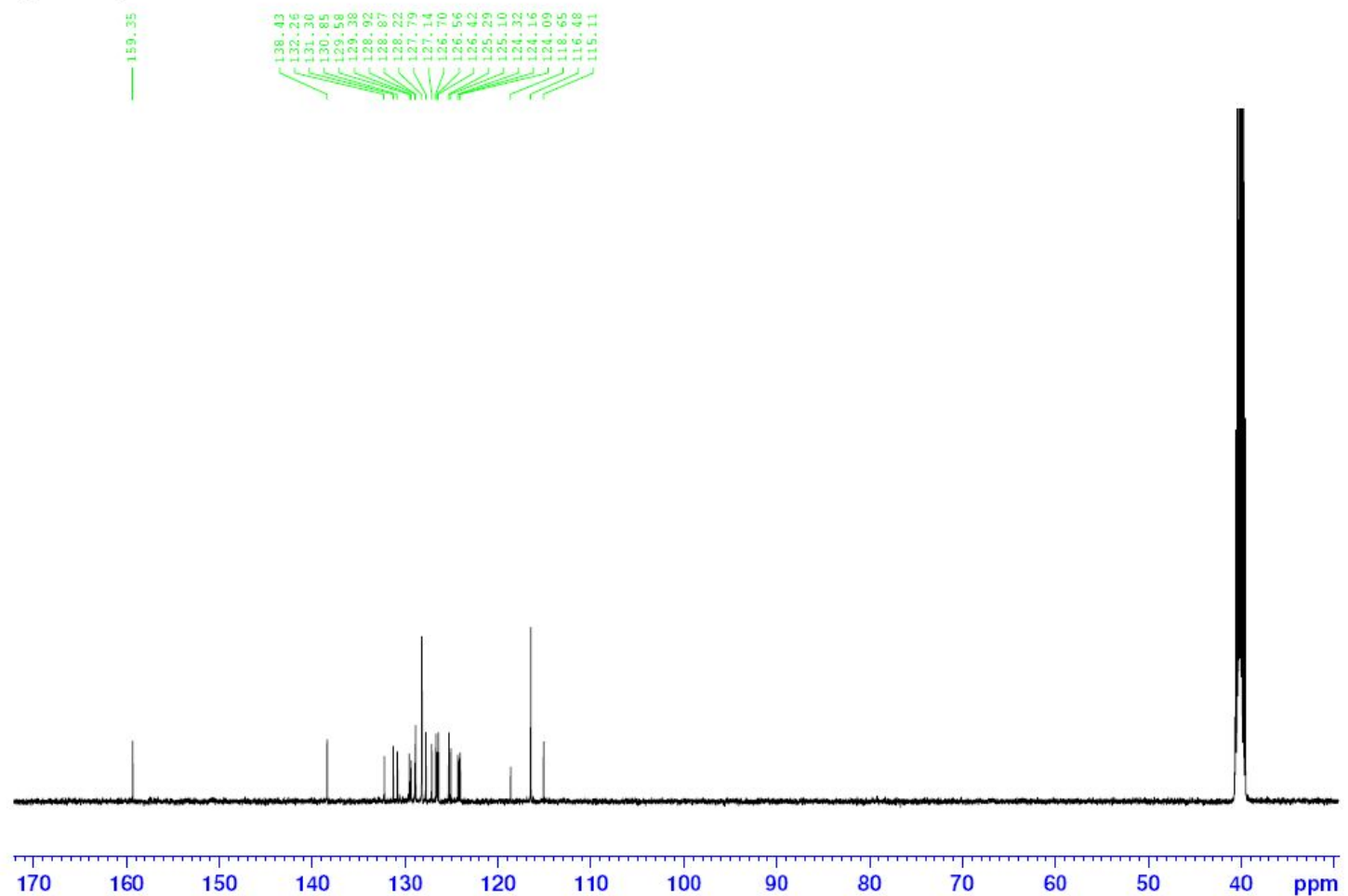
Fig.S20 ${ }^{1} \mathrm{H}$ and ${ }^{13} \mathrm{C}$ NMR spectrum of monohydroxystilbene 7
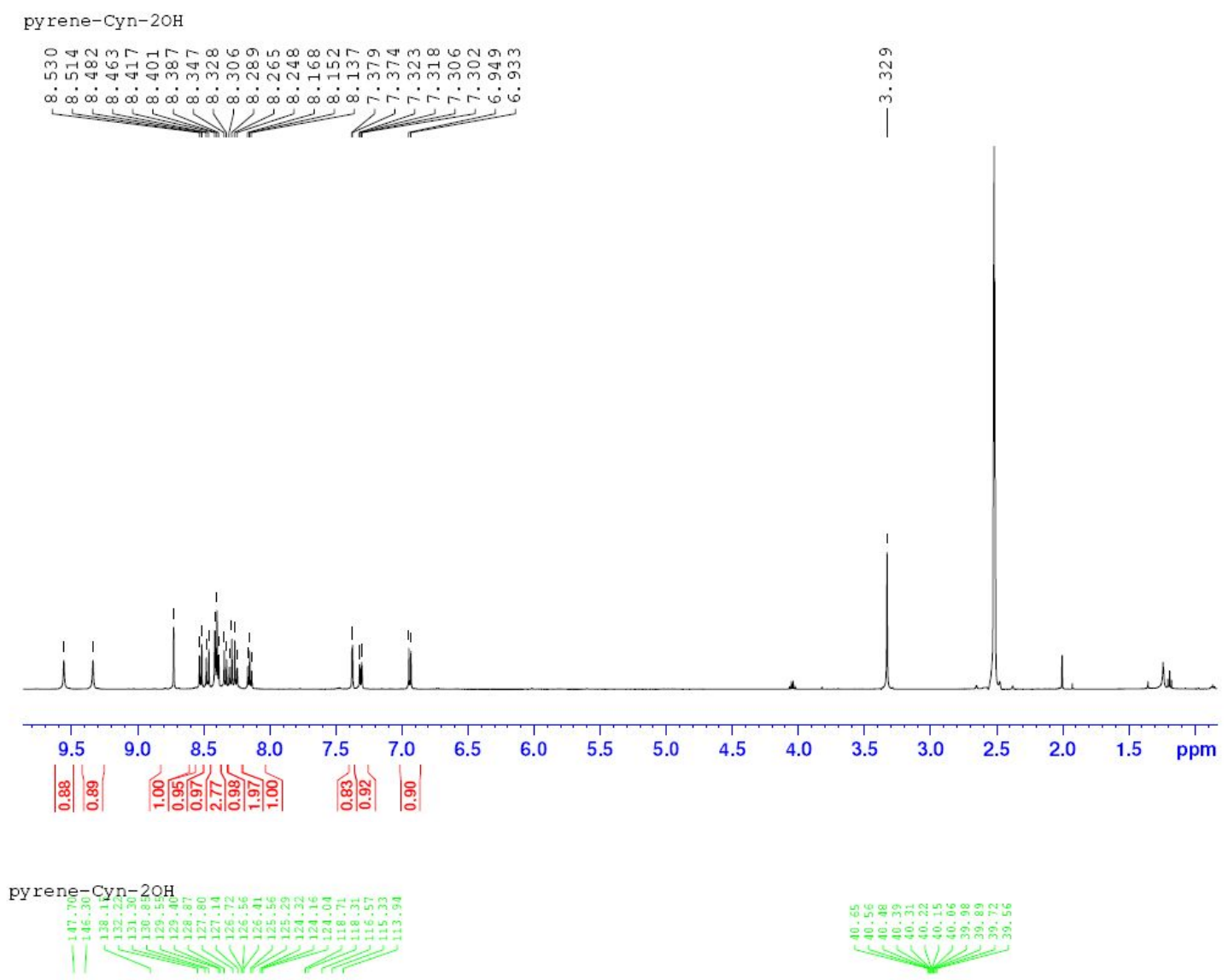

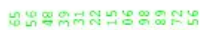

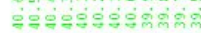

vivise

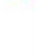
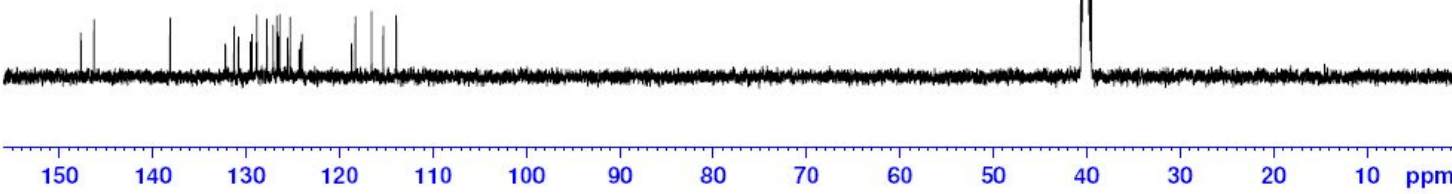

Fig.S21 ${ }^{1} \mathrm{H}$ and ${ }^{13} \mathrm{C}$ NMR spectrum of dihydroxystilbene 8 


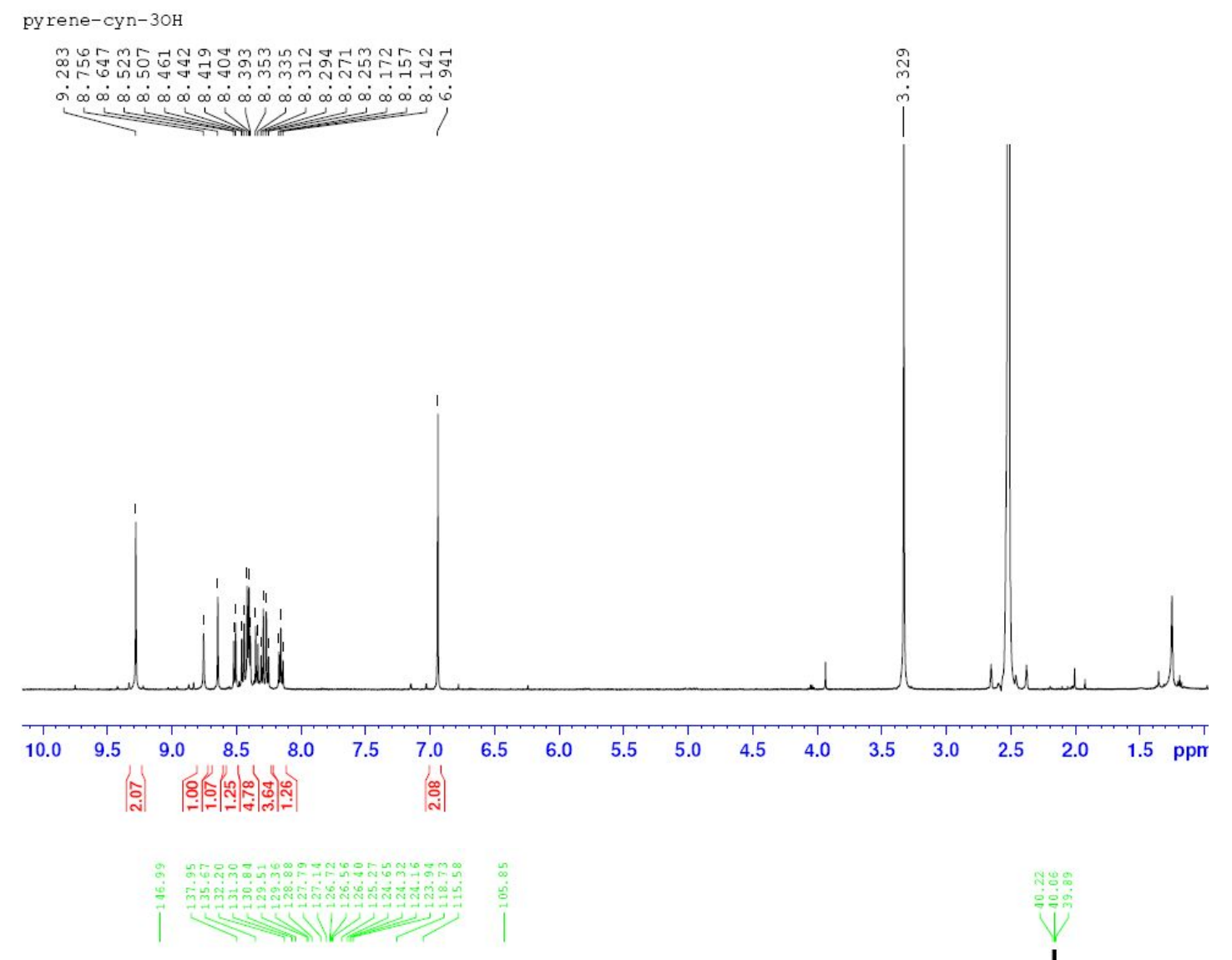

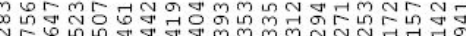

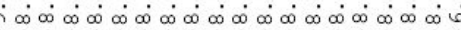

(
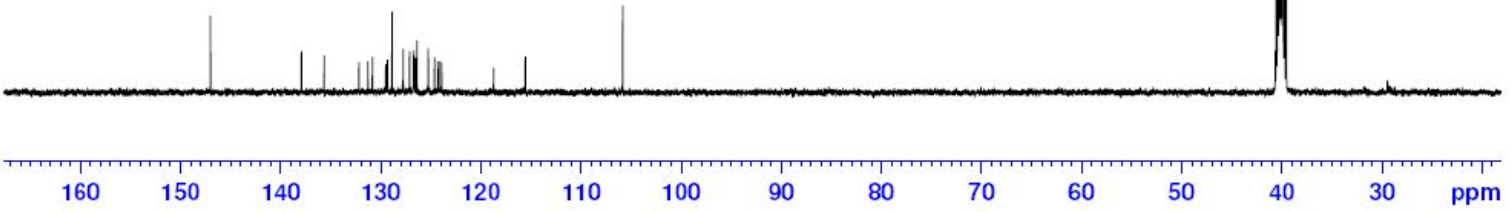

Fig.S22 ${ }^{1} \mathrm{H}$ and ${ }^{13} \mathrm{C}$ NMR spectrum of trihydroxystilbene 9 


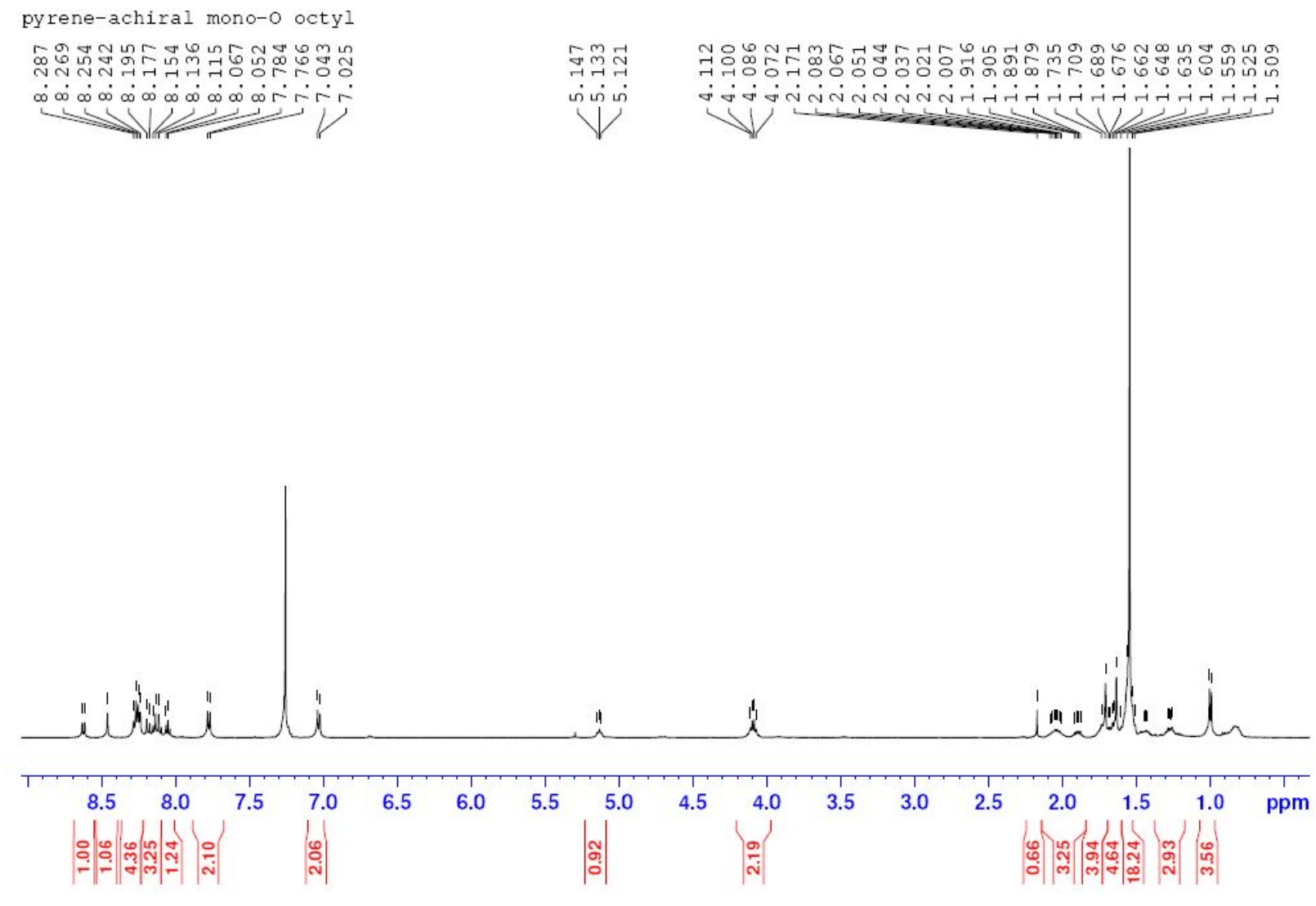

achiral mono pyrene octyl

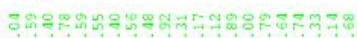

m.

$\sim^{2}$

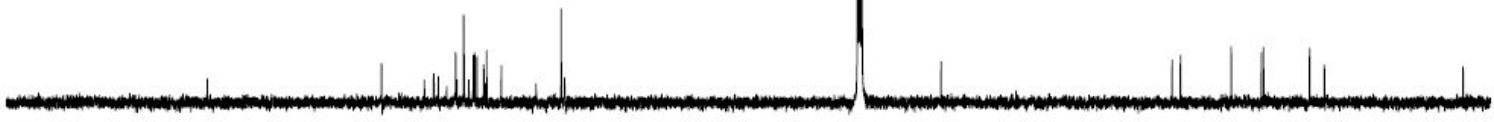

\begin{tabular}{|c|c|c|c|c|c|c|c|c|c|c|c|c|c|c|c|c|}
\hline 180 & 170 & 160 & 150 & 140 & 130 & 120 & 110 & 100 & 90 & 80 & 70 & 60 & 50 & 40 & 30 & 20 \\
\hline
\end{tabular}

Fig.S23 ${ }^{1} \mathrm{H}$ and ${ }^{13} \mathrm{C}$ NMR spectrum of MA 1 
pyrene-mono-cyn-octyl chiral

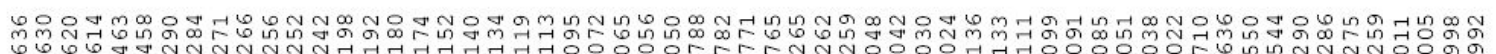

1.

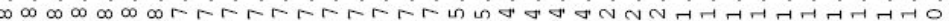

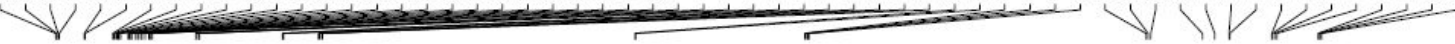

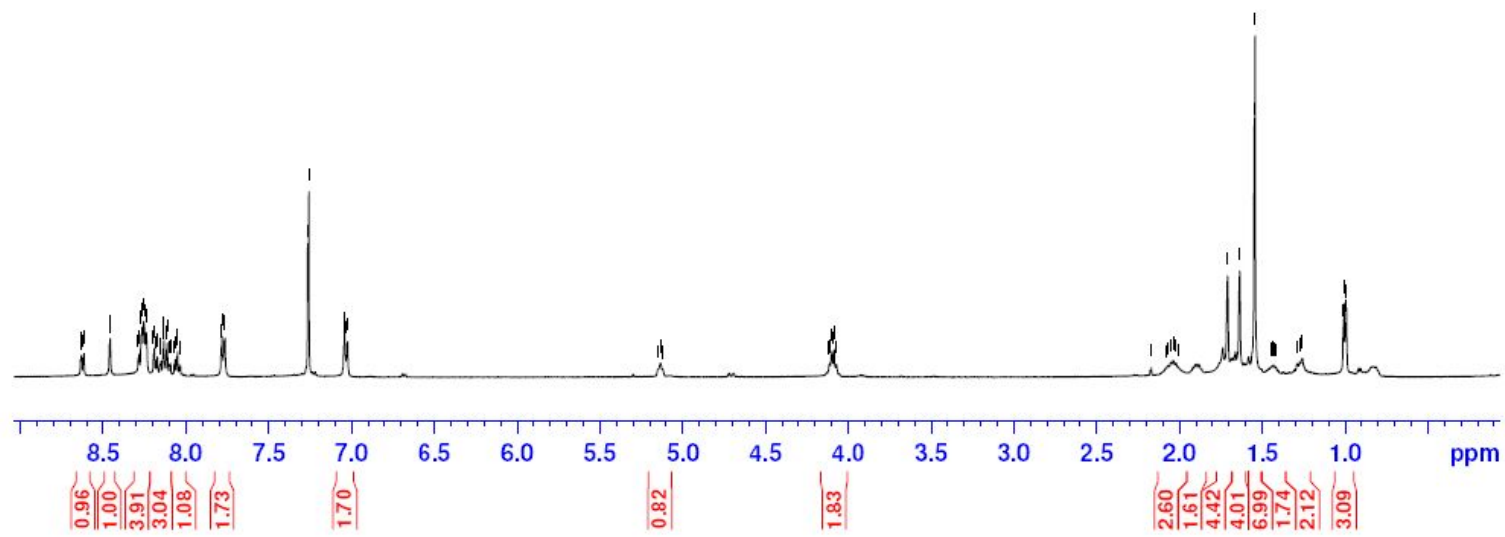

pyrene-mono chiral octa $13 \mathrm{C}$

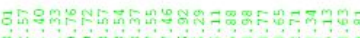

1

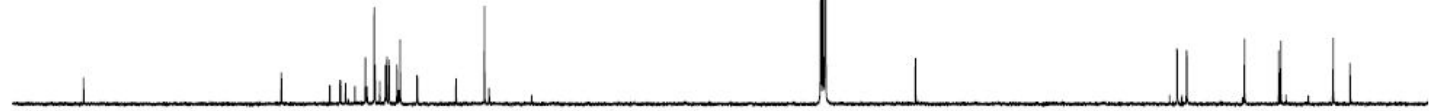

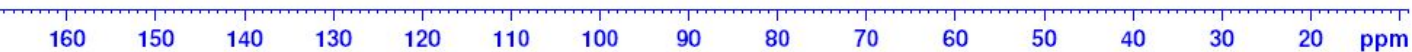

Fig.S24 ${ }^{1} \mathrm{H}$ and ${ }^{13} \mathrm{C}$ NMR spectrum of MC 2 


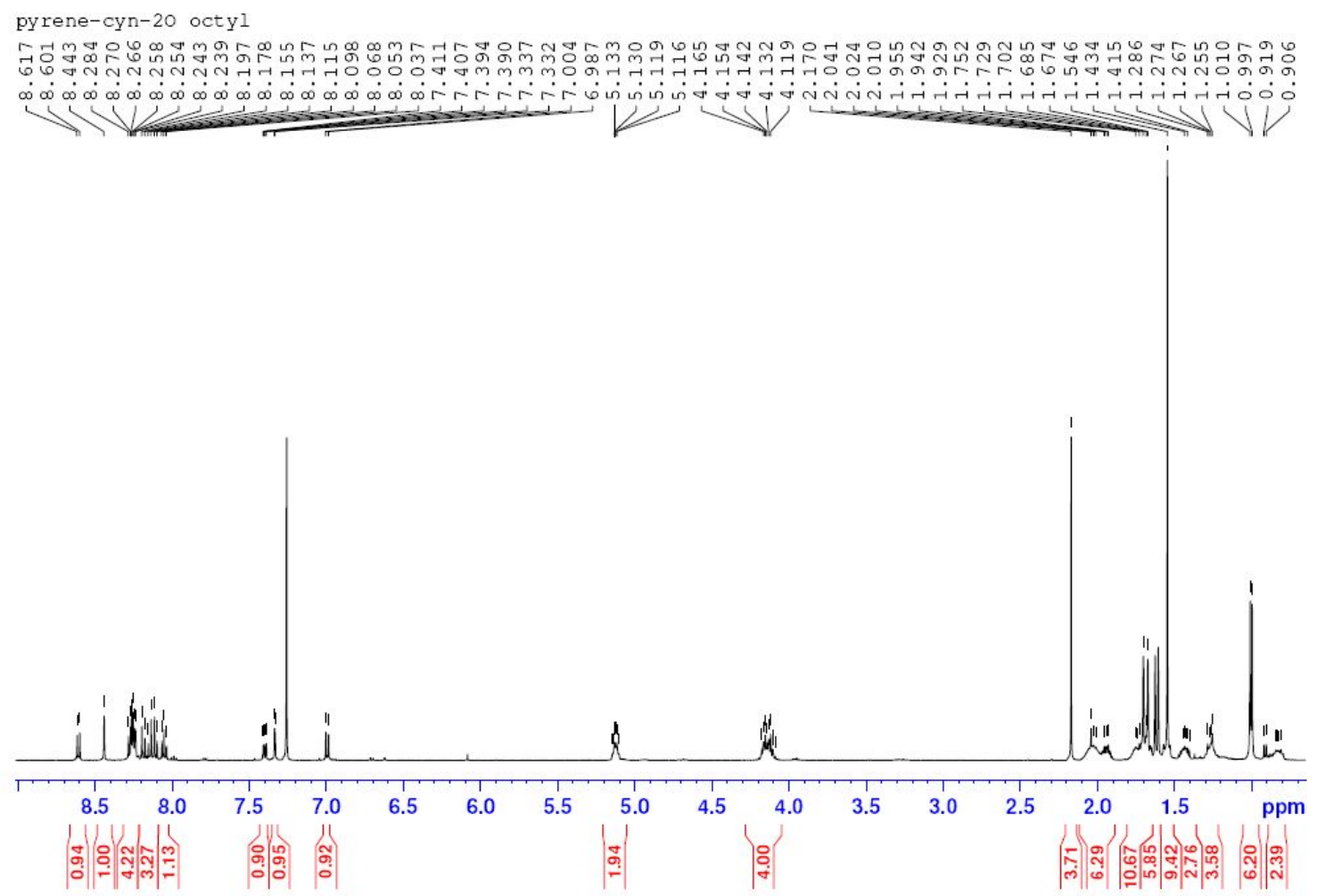

pyrene- di-o -octyl achiral

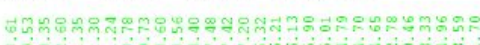

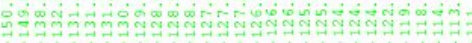

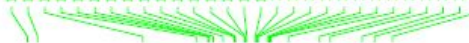

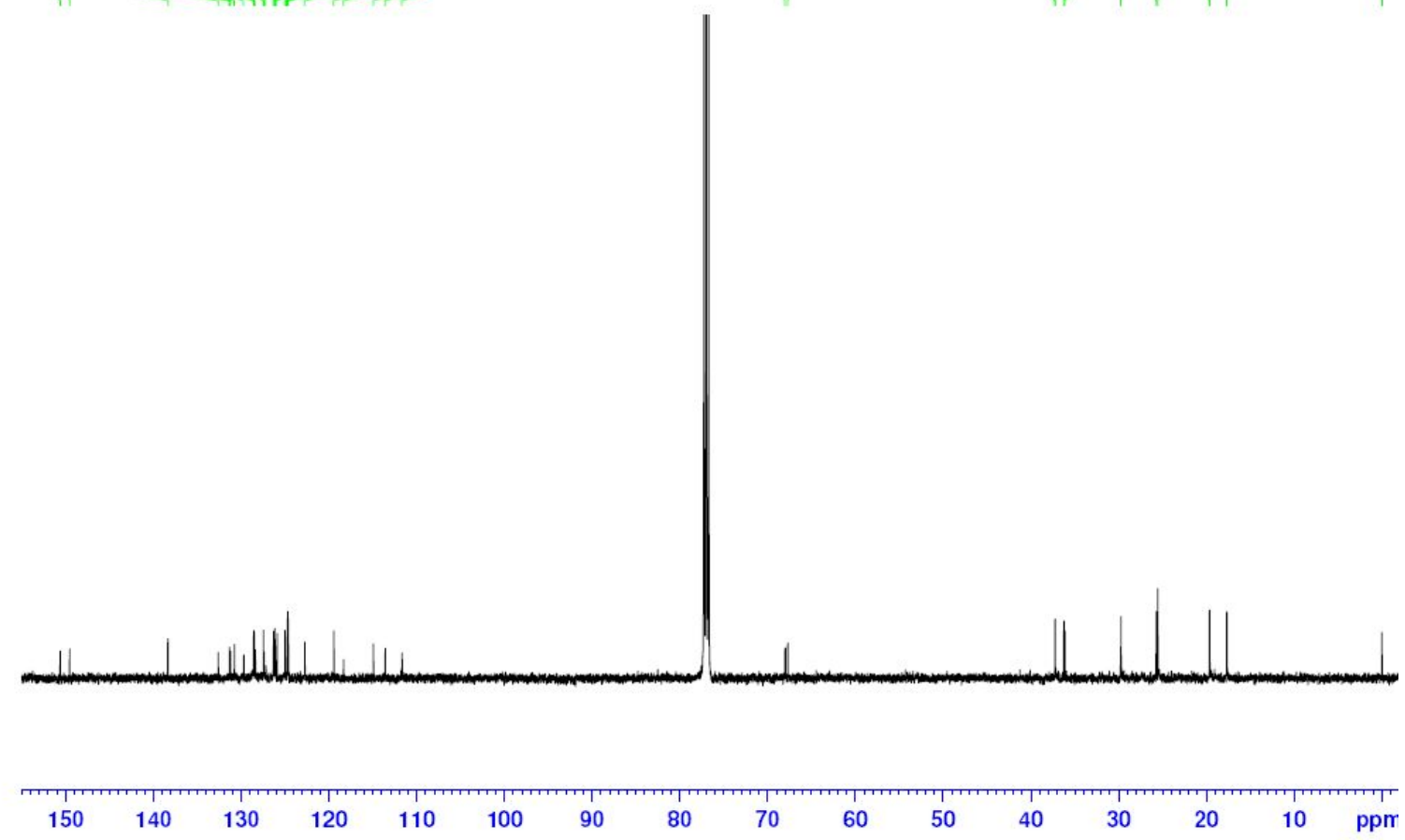

Fig.S25 ${ }^{1} \mathrm{H}$ and ${ }^{13} \mathrm{C}$ NMR spectrum of DA 3 


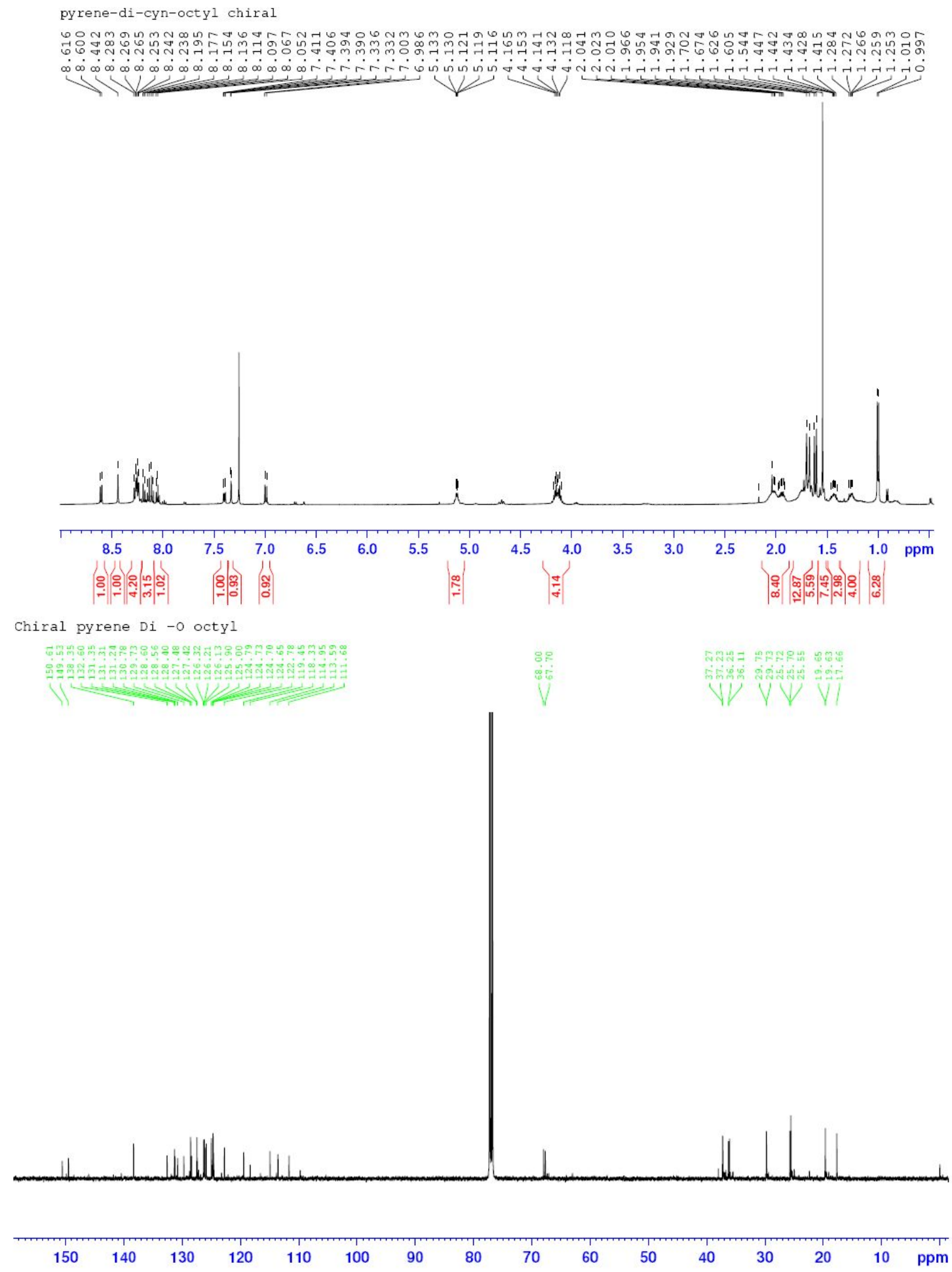

Fig.S26 ${ }^{1} \mathrm{H}$ and ${ }^{13} \mathrm{C}$ NMR spectrum of DC 4 
pyrene-achiral tri-o-octyl

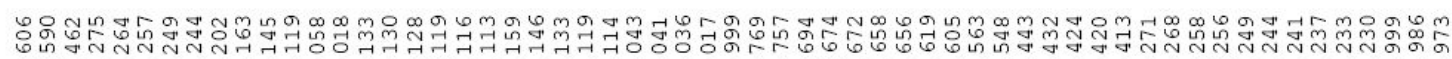

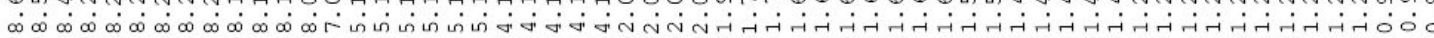

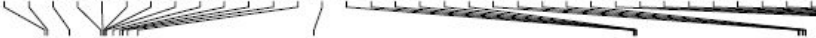
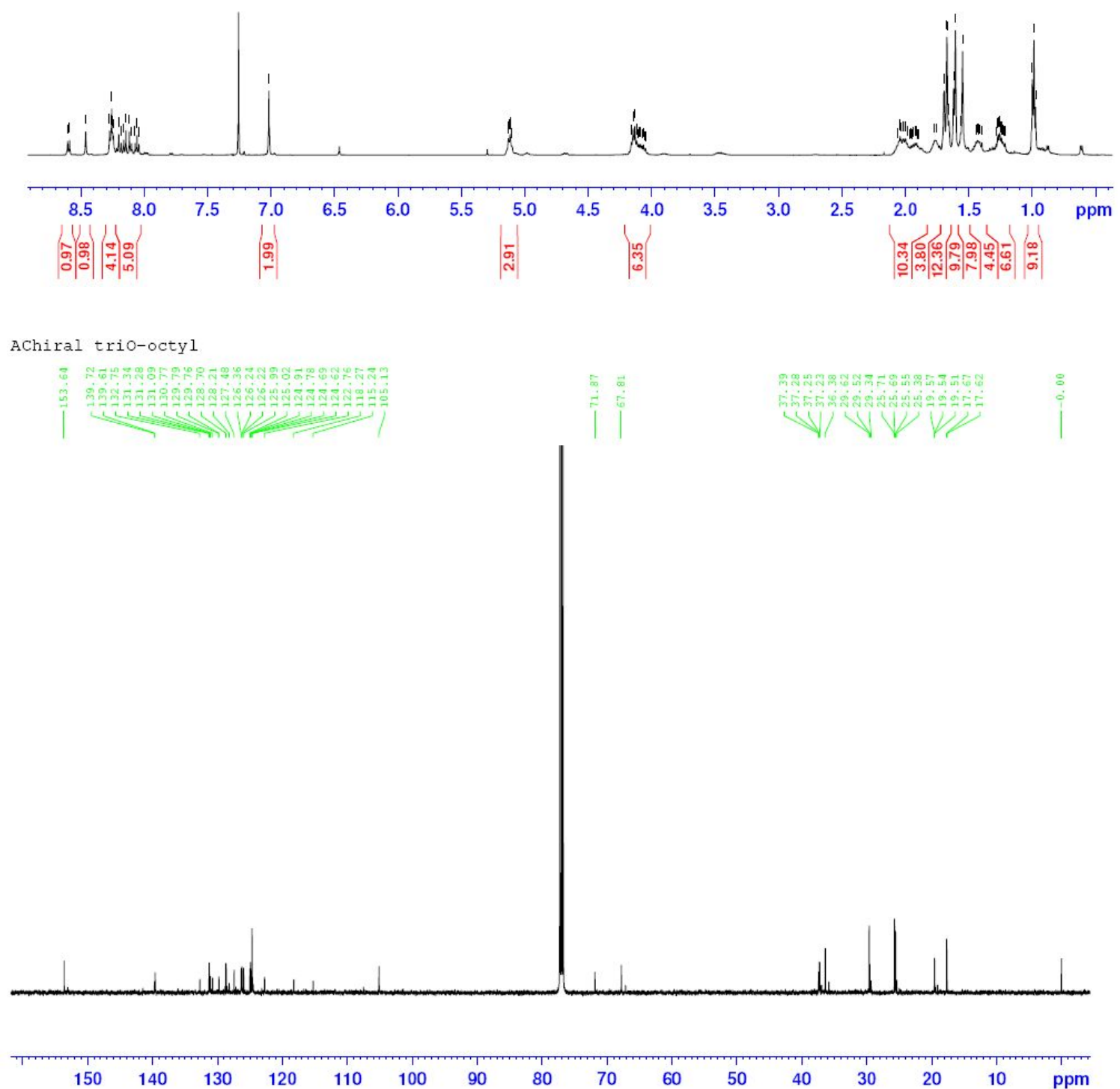

Fig. S2 $7{ }^{1} \mathrm{H}$ and ${ }^{13} \mathrm{C}$ NMR spectrum of TA 5 
Pyrene-tri-o octyl chiral

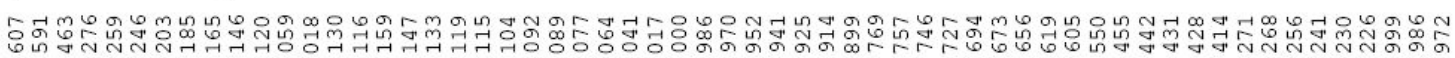

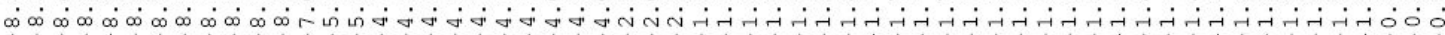

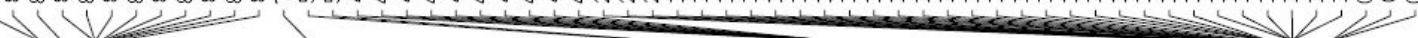
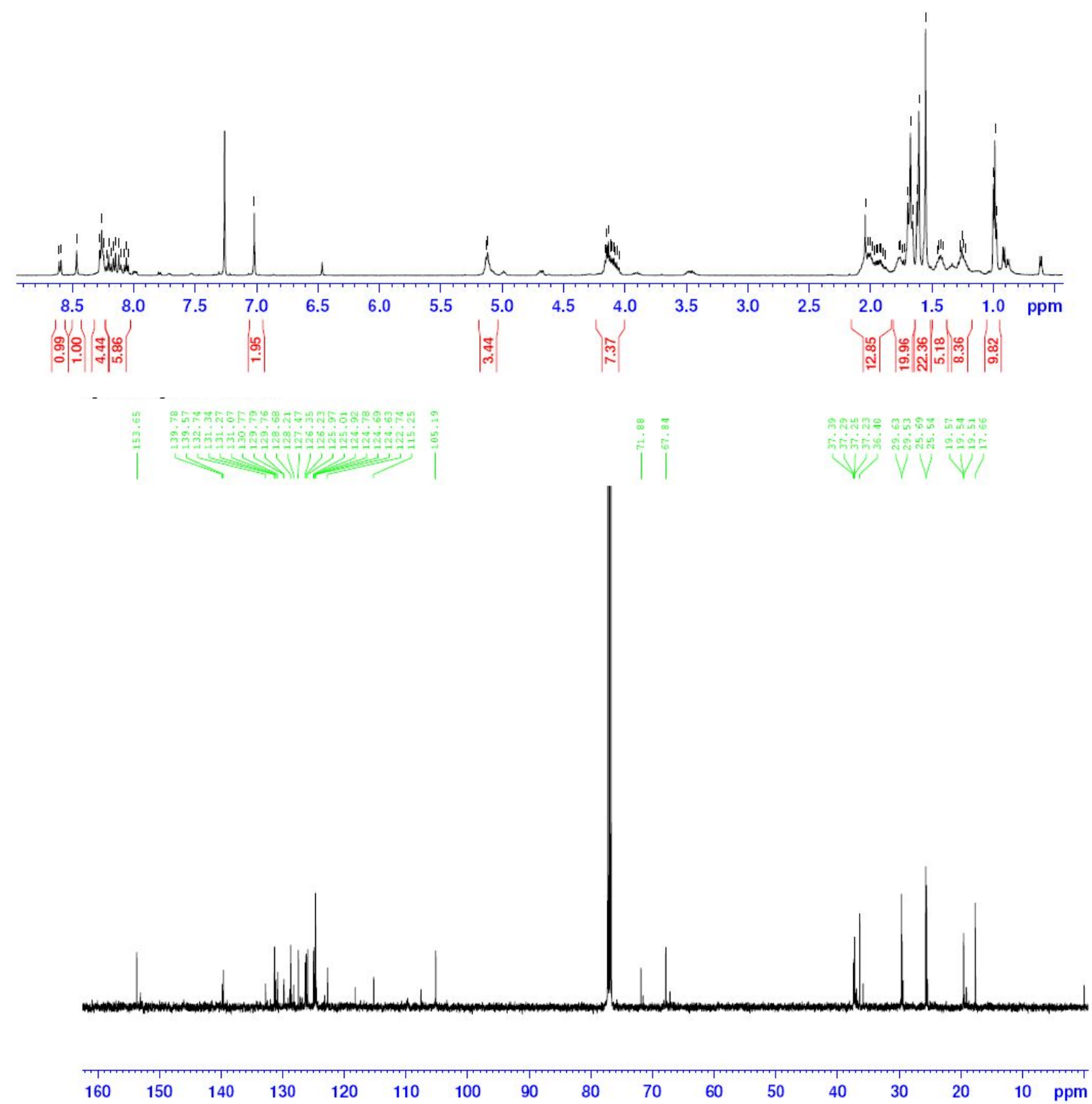

Fig. S28 ${ }^{1} \mathrm{H}$ and ${ }^{13} \mathrm{C}$ NMR spectrum of TC 6. 